\title{
UNIFORM ASYMPTOTIC ESTIMATES OF TRANSITION PROBABILITIES ON COMBS
}

\author{
DANIELA BERTACCHI and FABIO ZUCCA
}

(Received 20 November 2000; revised 14 August 2002)

Communicated by V. T. Stefanov

\begin{abstract}
We investigate the asymptotical behaviour of the transition probabilities of the simple random walk on the 2-comb. In particular, we obtain space-time uniform asymptotical estimates which show the lack of symmetry of this walk better than local limit estimates. Our results also point out the impossibility of getting sub-Gaussian estimates involving the spectral and walk dimensions of the graph.
\end{abstract}

2000 Mathematics subject classification: primary $60 \mathrm{~J} 10$.

Keywords and phrases: uniform estimate, uniform Lebesgue theorem, Green function, transition probability, Cauchy integral, comb.

\section{Introduction}

Given a random walk $\left(Z_{n}\right)_{n \geq 0}$ on a graph $X$, there are many related questions regarding its behaviour when the discrete time parameter $n$ goes to infinity. Classical questions of this kind are, for instance: will the random walk visit a given vertex of the graph only a finite number of times (with probability one)? Will it leave any bounded set after a finite time (with probability one)? Moreover, if we denote by $p^{(n)}(x, y)$ the $n$-step probabilities of the random walk from the vertex $x$ to the vertex $y$ of $X$, we can study some features of the sequence $\left(p^{(n)}(x, y)\right)_{n}$, for instance, answer to the question: is it asymptotic to some 'nice' numerical sequence?

Answers to the first two questions are theorems and criteria for recurrence and transience; answers to the latter question are provided by local limit theorems, that is, theorems which give a numerical estimate of $p^{(n)}(x, y)$ for fixed $x, y$ as $n$ tends to infinity. Local limit theorems are widely studied (in many papers in literature) in 


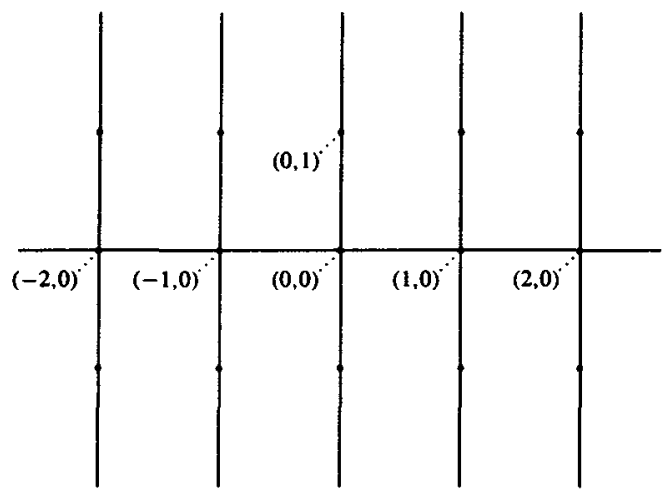

FIGURE 1. The 2-comb.

various settings: for instance, the case of random walks on free products of discrete groups was studied by Woess [17] and Cartwright and Soardi [5] (see also [4] or [6] in the case of Cartesian product of discrete groups).

The present work studies the asymptotic behaviour of the transition probabilities of the simple random walk on the 2-comb, which is a graph obtained attaching at each point of $\mathbb{Z}$ another copy of $\mathbb{Z}$ by its origin (see Figure 1 ).

A local limit theorem for the 2-comb (and in general for $d$-dimensional combs) and $x=y$ is well known: here we observe how to extend it to the asymptotic estimate of $\left(p^{(n)}(x, y)\right)_{n}$ for any $x$ and $y$ (equation (3.3)). The simple random walk on the 2-comb lacks symmetry (more precisely it is not isotropic-see [3]) and this feature is not shown by local limit estimates. To stress the different behaviour the random walk has in the two principal directions (vertical and horizontal) one needs space-time estimates.

A space-time asymptotic estimate is a result which provides an estimate of $p^{(n)}(x, y)$ as $n$ tends to infinity, uniform with respect to the quotient $d(x, y) / n$ lying in a suitable range. Of course local limit theorems can be derived from space-time estimates. We provide space-time asymptotic estimates for the $p^{(n)}(x, y)$ when $y=o:=(0,0)$ and $x=(k, 0)$ or $x=(0, k)$, that is, results of the form

$$
p^{(n)}(x, o) \stackrel{n}{\sim} C f\left(\frac{d(x, o)}{n}, n\right) n^{-d}
$$

where $C, d$ are constants and $f$ is a real valued function which all depend on the range of $d(x, o) / n$. From these results all known limit theorems for the 2-comb can be derived as a particular case.

The technique we exploit is essentially a Laplace-type estimate of integrals, since the transition probabilities can be written as integrals thanks to the Cauchy formula 
for the coefficients of the power series of an holomorphic function:

$$
p^{(n)}(x, o)=\frac{1}{2 \pi i} \int_{\gamma} \frac{G(x, o \mid z)}{z^{n+1}} d z
$$

where $G(x, o \mid z)$ is the Green function (see (3.1)) associated with the walk and has an explicit expression, and $\gamma$ is a positively oriented, simple closed curve in $\mathbb{C}$ surrounding 0 . The basic idea is that the main part of the integral is given by integration on the part of the curve which is closer to the singularity $z=1$ of $G(x, o \mid z)$. To develop this idea into mathematical terms we first separate in the integrand the part with algebraic behaviour and the part with exponential behaviour, then we choose a suitable curve of integration and its parametrization. Afterwards, we write the Taylor expansion of the argument of the exponential part of the integrand as a function of the parameter of the curve and we finally show that it is possible to choose a piece of the curve on which integration gives the asymptotic behaviour of the transition probabilities. It is remarkable that the above mentioned Taylor expansions are very different in the two cases $x=(k, 0)$ and $x=(0, k)$. This results in two different asymptotic behaviours of the transition probabilities when $d(x, o) / n$ tends to 0 : in the first case

$$
p^{(n)}(x, o) \stackrel{n}{\sim} c_{1} \exp \left(c_{2} n(d(x, o) / n)^{4 / 3}\right) n^{-3 / 4},
$$

while in the second case

$$
p^{(n)}(x, o) \stackrel{n}{\sim} c_{3} \exp \left(c_{4} n(d(x, o) / n)^{2}\right) n^{-3 / 4} .
$$

In particular, this shows that for the 2-comb it is impossible to give sub-Gaussian estimates of the transition probabilities (see Section 10); the 2-comb seems to be the simplest graph for which this happens.

We give a brief outline of the paper. In Section 2 we list the definition of uniform estimate with respect to a parameter and two Lebesgue-type theorems which are needed to obtain such estimates. Section 3 recalls local limit theorems and generating functions for combs. Then in Section 4 and Section 5 uniform estimates are proved for the vertical direction, while in Section 6, Section 7, Section 8 and Section 9 we prove uniform estimates for the horizontal direction. In the last section we discuss the obtained results and possible extensions.

\section{Asymptotic estimates: definitions and technical results}

In this paper we are concerned with asymptotic estimates of transition probabilities. We then give some useful definitions and theorems. The first definition extends the usual definition of asymptotic sequences (let $X$ be a one point space and $A_{n} \equiv X$ for all $n \in \mathbb{N}$ ). 
DEFINITION 2.1. Given two sequences $\left(a_{n}(x)\right)_{n}$ and $\left(b_{n}(x)\right)_{n}$ of complex functions defined on a space $X$ and a sequence $\left(A_{n}\right)_{n}$ of subsets of $X$, we say that $a_{n}$ is asymptotic to $b_{n}$ (and we write $a_{n} \stackrel{n}{\sim} b_{n}$ ) as $n$ tends to infinity, uniformly with respect to $x \in A_{n}$ if and only if there exists a sequence of complex functions $\left(o_{n}(x)\right)_{n}$ and $n_{0} \in \mathbb{N}$ such that:

(i) $a_{n}(x)=b_{n}(x)\left(1+o_{n}(x)\right)$ for every $n \geq n_{0}, x \in A_{n}$;

(ii) for every $\varepsilon>0$ there exists $n_{\varepsilon} \in \mathbb{N}, n_{\varepsilon} \geq n_{0}$, such that for every $n>n_{\varepsilon}$, $x \in A_{n}$ we have $\left|o_{n}(x)\right|<\varepsilon$.

The following definition extends the definition of uniform convergence (take $A_{n}=$ $X$ for all $n \in \mathbb{N}$ ).

DEFINITION 2.2. Let us consider a sequence $\left(a_{n}(x)\right)_{n}$ of functions defined on a space $X$ with values on a metric space $(Y, d)$ and let $\left(A_{n}\right)_{n}$ be a sequence of subsets of $X$. Let $b: X \rightarrow Y$, we say that $\left(a_{n}\right)_{n}$ converges to $b$ when $n$ tends to infinity, uniformly with respect to $x \in A_{n}$ if and only if for every $\varepsilon>0$ there exists $n_{\varepsilon} \in \mathbb{N}$ such that for every $n \geq n_{\varepsilon}, x \in A_{n}$ we have $d\left(a_{n}(x), b(x)\right)<\varepsilon$.

The following two technical results are the tools which we use to deal with integrals of sequences and their uniform convergence. The first theorem provides an extension of Lebesgue's bounded convergence theorem to the case of uniform convergence (with respect to Definition 2.2).

THEOREM 2.3. Let $(X, \Sigma, \mu)$ be a complete measure space, $f_{n}: X \times Y \rightarrow \mathbb{C}$, for all $n \in \mathbb{N}$, and $f: X \times Y \rightarrow \mathbb{C}$. Let $\left(A_{n}\right)_{n}$ be a sequence of subsets of $Y$. Suppose that

(a) $f_{n}$ is measurable with respect to $x \in X$ for every fixed $y \in Y$;

(b) for every $y \in Y, f_{n}(x, y) \stackrel{n \rightarrow \infty}{\longrightarrow} f(x, y)$, $\mu$-a.e.;

(c) $f_{n}(x, y) \stackrel{n \rightarrow \infty}{\longrightarrow} f(x, y)$, uniformly with respect to $y \in A_{n}, \mu$-a.e.;

(d) there exists $g \in L^{1}(\mu)$ such that $|f(x, y)| \leq g(x),\left|f_{n}(x, y)\right| \leq g(x)$, $\mu$-a.e., for every $y \in A_{n}, n \in \mathbb{N}$.

Then $f(\cdot, y)$ is measurable for all $y \in Y$ and $f_{n}(\cdot, y) \rightarrow f(\cdot, y)$ in $L^{1}(\mu)$ uniformly with respect to $y \in A_{n}$.

PROOF. Measurability of $f(\cdot, y)$ is a standard fact. Let us fix $\varepsilon>0$. Since $g \in L^{1}(\mu)$, there exists $X(\varepsilon) \in \Sigma$ such that $\mu(X(\varepsilon))<\infty$ and $\int_{X(\varepsilon)} g d \mu<\varepsilon$, and there is $\delta_{\varepsilon}>0$ such that $\int_{E} g d \mu<\varepsilon$ if $E \in \Sigma$ and $\mu(E)<\delta_{\varepsilon}$.

For $n \in \mathbb{N}$, let

$$
X_{n}(\varepsilon)=\left\{x \in X(\varepsilon):\left|f_{m}(x, y)-f(x, y)\right|<\varepsilon / \mu(X(\varepsilon)) \text { for all } y \in A_{m}, m \geq n\right\}
$$


Clearly, $X_{n}(\varepsilon) \subset X_{n+1}(\varepsilon)$, and $\mu\left(X(\varepsilon) \backslash \bigcup_{n} X_{n}(\varepsilon)\right)=0$. Thus we can find $n_{\varepsilon}$ such that $\mu\left(X(\varepsilon) \backslash X_{n}(\varepsilon)\right)<\delta_{\varepsilon}$ for all $n \geq n_{\varepsilon}$. Then for $y \in A_{n}$ and $n \geq n_{\varepsilon}$,

$$
\begin{aligned}
& \int_{X}\left|f_{n}(x, y)-f(x, y)\right| d \mu \\
& \quad=\int_{X(\varepsilon)^{c}}\left|f_{n}(x, y)-f(x, y)\right| d \mu+\int_{X(\varepsilon)}\left|f_{n}(x, y)-f(x, y)\right| d \mu \\
& \quad \leq 2 \int_{X \backslash X_{n}(\varepsilon)} g d \mu+\int_{X_{n}(\varepsilon)}\left|f_{n}(x, y)-f(x, y)\right| d \mu<5 \varepsilon .
\end{aligned}
$$

Sometimes it will be impossible to exhibit a limit function for our estimates, but we will be able to find a sequence of functions asymptotic to the given one and much simpler. In that direction, the following theorem is useful.

THEOREM 2.4. Let $(X, \Sigma, \mu)$ be a measure space and let $\left(f_{n}\right)_{n},\left(h_{n}\right)_{n},\left(o_{n}\right)_{n}$ be three sequences of complex-valued functions defined on $X \times Y$ which are measurable with respect to $x \in X$ for every fixed $y \in Y$. Let $\left(A_{n}\right)_{n}$ be a sequence of subsets of $Y$. Suppose that

(a) $h_{n}(x, y)=f_{n}(x, y)\left(1+o_{n}(x, y)\right)$, $\mu$-a.e., for all $y \in A_{n}, n \in \mathbb{N}$;

(b) $o_{n}(x, y) \stackrel{n \rightarrow \infty}{\longrightarrow} 0 \mu$-a.e., uniformly with respect to $y \in A_{n}$;

(c) there exist $g \in L^{1}(\mu)$ such that $\left|h_{n}(x, y)\right| \leq g(x),\left|f_{n}(x, y)\right| \leq g(x)$, $\mu$-a.e., for all $y \in A_{n}, n \in \mathbb{N}$;

(d) there exists $c>0$ such that $\left|\int_{X} f_{n}(x, y) d \mu\right| \geq c$, for all $y \in A_{n}, n \in \mathbb{N}$.

Then

$$
\int_{X} f_{n}(x, y) d \mu \stackrel{n}{\sim} \int_{X} h_{n}(x, y) d \mu
$$

uniformly with respect to $y \in A_{n}$.

Proof. Let us fix $\epsilon>0$ and define $X_{n}(\epsilon)=\left\{x \in X:\left|o_{m}(x, y)\right|<\epsilon\right.$, for all $m \geq$ $\left.n, y \in A_{m}\right\}$. By the hypotheses, we have

$$
\begin{aligned}
\left|1-\frac{\int_{X} h_{n}(x, y) d \mu}{\int_{X} f_{n}(x, y) d \mu}\right| \leq & \frac{\left|\int_{X_{n}(\varepsilon)^{r}} f_{n}(x, y) d \mu\right|}{\left|\int_{X} f_{n}(x, y) d \mu\right|} \\
& +\frac{\left|\int_{X_{n}(\varepsilon)} f_{n}(x, y) d \mu-\int_{X} h_{n}(x, y) d \mu\right|}{\left|\int_{X} f_{n}(x, y) d \mu\right|} \\
& \leq c^{-1}\left(2 \int_{X_{n}(\varepsilon)^{r}} g d \mu+\epsilon \int_{X} g d \mu\right),
\end{aligned}
$$

for all $y \in A_{n}, n \in \mathbb{N}$. Since $\mu\left(X_{n}(\varepsilon)^{c}\right) \rightarrow 0$ as $n \rightarrow \infty$, the theorem is proved. 
The last (non-standard) lemma deals with the triangular inequality for power series: we are interested in the cases where a strict inequality holds.

LEMMA 2.5. Let $f(z)=\sum_{n=0}^{\infty} a_{n} z^{n}$ be a power series with positive radius of convergence $R$, and with $a_{n} \geq 0$ for all $n$. Then $|f(z)| \leq f(|z|)$ whenever $|z|<R$. If there exists $n \in \mathbb{N}$ such that $a_{n}>0$ and $a_{n+1}>0$, then $|f(z)|<f(|z|)$ unless $z=|z|$.

PROOF. The inequality $|f(z)| \leq f(|z|)$ is obvious. If equality holds for some $z$, then there is a $\theta \in \mathbb{R}$ such that $a_{n} z^{n}=\left|a_{n} z^{n}\right| e^{i \theta}$ for all $n$. If $a_{n}>0$ and $a_{n+1}>0$, then $z^{n}=\left|z^{n}\right| e^{i \theta}$ and $z^{n+1}=\left|z^{n+1}\right| e^{i \theta}$. Dividing the second equation by the first, we get $z=|z|$.

\section{Local limit theorems and generating functions}

The 2-comb lattice $C_{2}$ is a spanning tree of $\mathbb{Z}^{2}$, that is, a subgraph of $\mathbb{Z}^{2}$ which is a tree and contains all vertices. Thus there is a natural choice of coordinates on the comb (that is, $(x, y) \in C_{2}$ indicates the same point of $\mathbb{Z}^{2}$, now thought as belonging to $C_{2}$ ).

More generally, $d$-dimensional comb lattices $C_{d}$ are the spanning trees of $\mathbb{Z}^{d}$ obtained inductively by attaching at each point of $C_{d-1}$ a copy of $\mathbb{Z}$.

The estimate of the asymptotic behaviour of the transition probabilities of the simple random walk on comb lattices passes through the knowledge of the generating functions, which we now recall.

Recall that $p^{(n)}(x, y)$ is defined as the probability that the random walk starting at $x$ is in $y$ at time $n$. The Green function is then the power series

$$
G(x, y \mid z)=\sum_{n=0}^{\infty} p^{(n)}(x, y) z^{n}, \quad z \in \mathbb{C},
$$

while

$$
F(x, y \mid z)=\sum_{n=0}^{\infty} f^{(n)}(x, y) z^{n}, \quad z \in \mathbb{C},
$$

where $f^{(n)}(x, y)$ is the probability that the random walk starting at $x$ reaches $y$ for the first time at time $n$. Then it is well known (see for instance, [18, Lemma 1.13]) that in the common domain of convergence of these power series $G(x, y \mid z)=$ $G(y, y \mid z) F(x ; y \mid z)$.

At least when $x=y=o$, the Green function $G_{d}$ of the $d$-dimensional comb can be obtained recursively by the following formula (see $[8,7]$ )

$$
G_{d}(o, o \mid z)=\frac{d}{\sqrt{\left(1+(d-1) / G_{d-1}(z)\right)^{2}-z^{2}}},
$$


recalling that $G_{1}(o, o \mid z)=1 / \sqrt{1-z^{2}}$ (note that we will use no subscripts when $d=2)$.

From (3.2), using techniques which can be found in [2], one can obtain local limit estimates for the transition probabilities

$$
p^{(2 n)}(o, o) \stackrel{n}{\sim} \frac{2^{2^{-d+1}}}{\Gamma\left(2^{-d}\right)} n^{2^{-d}-1},
$$

$\left(p^{(2 n+1)}(o, o)=0\right.$ for all $\left.n\right)$. This was done by Weiss and Havlin [10] in the case $d=2,3$, and in the general case by Gerl [8] and Cassi and Regina [7].

From this particular estimate one can easily derive an estimate of the general transition probabilities (apply the results of [3, Section 6])

$$
p^{(n)}(x, y) \stackrel{n}{\sim} \frac{2^{2^{-d}-1} \operatorname{deg}(y)}{\Gamma\left(2^{-d}\right)} n^{2^{-d}-1},
$$

where $n+d(x, y)$ is even $\left(p^{(n)}(x, y)=0\right.$ if $n+d(x, y)$ is odd).

In (3.3) $x$ and $y$ are fixed: our goal is to obtain asymptotic estimates of $p^{(n)}\left(x_{k}, o\right)$ and of $p^{(n)}\left(y_{k}, o\right)$, where $x_{k}=(k, 0), y_{k}=(0, k), k \geq 0$, uniform with respect to the parameter $\xi=k / n$ (in order to avoid discussions about the parity of $n$ and $k$ we will only deal with the case where they both are even, but this is no severe restriction-see Section 4 and Section 10).

Since we are going to use formula (1.1), we need the explicit expressions of $G\left(x_{k}, o \mid z\right)$ and $G\left(y_{k}, o \mid z\right)$

$$
\begin{aligned}
& G\left(x_{k}, o \mid z\right)=\frac{\sqrt{2}}{\sqrt{1-z^{2}+\sqrt{1-z^{2}}}}\left(\frac{1+\sqrt{1-z^{2}}-\sqrt{2} \sqrt{1-z^{2}+\sqrt{1-z^{2}}}}{z}\right)^{|k|} \\
& G\left(y_{k}, o \mid z\right)=\frac{\sqrt{2}}{\sqrt{1-z^{2}+\sqrt{1-z^{2}}}}\left(\frac{1-\sqrt{1-z^{2}}}{z}\right)^{|k|} .
\end{aligned}
$$

The computation uses well-known techniques for the generating functions on graphs involving explicit expressions of $G(o, o \mid z), F\left(x_{k}, o \mid z\right)$ and $F\left(y_{k}, o \mid z\right)$ (an example of these techniques in the case of the homogeneous tree is [18, Lemma 1.24]).

For simplicity we denote by

$$
\begin{aligned}
& G(z):=G(o, o \mid \sqrt{z})=\frac{\sqrt{2}}{\sqrt{1-z+\sqrt{1-z}}} ; \\
& F_{1}(z):=F\left(y_{1}, o \mid \sqrt{z}\right)=\frac{1-\sqrt{1-z}}{\sqrt{z}} ; \\
& F_{2}(z):=F\left(x_{1}, o \mid \sqrt{z}\right)=\frac{1+\sqrt{1-z}-\sqrt{2} \sqrt{1-z+\sqrt{1-z}}}{\sqrt{z}} .
\end{aligned}
$$


The first steps in the direction of obtaining estimates for $p^{(2 n)}(x, o)$ are the same for $x=x_{k}$ and $x=y_{k}$, so we describe them here. We take $\gamma^{\prime}$ having $o$ in its interior and 1 in its exterior and we apply the substitution $u=z^{2}$ in (1.1). When $\gamma^{\prime}: z=z(t)$ describes one circuit about $o$ then $\gamma: u=u(t)$ describes two times the corresponding circuit, hence

$$
\begin{aligned}
& p^{(2 n)}\left(y_{2 k}, o\right)=\frac{1}{2 \pi i} \int_{\gamma} \frac{G(z) F_{1}(z)^{2 k}}{z^{n+1}} d z, \\
& p^{(2 n)}\left(x_{2 k}, o\right)=\frac{1}{2 \pi i} \int_{\gamma} \frac{G(z) F_{2}(z)^{2 k}}{z^{n+1}} d z .
\end{aligned}
$$

In order to stress the exponential part of the integrand, we write

$$
\frac{F_{i}(z)^{2 k}}{z^{n}}=\exp \left\{n \Psi_{\xi}^{i}(z)\right\}, \quad i=1,2,
$$

where $\xi:=k / n$ and

$$
\Psi_{\xi}^{i}(z)= \begin{cases}2 \xi \log (1-\sqrt{1-z})-(\xi+1) \log (z) & i=1 \\ 2 \xi \log (1+\sqrt{1-z}-\sqrt{2} \sqrt{1-z+\sqrt{1-z}})-(\xi+1) \log (z) & i=2\end{cases}
$$

Moreover, we will choose different curves of integration $\gamma$ with parametrization $z=z(\xi, t)$ and use this last substitution in (3.4). For simplicity we write

$$
\bar{\Psi}_{\xi}^{i}(t)=\Psi_{\xi}^{i}(z(\xi, t)), \quad i=1,2 .
$$

We note that the generating functions $G, F_{1}$ and $F_{2}$ all contain radicals, hence we must pay attention to their polidromy.

REMARK 3.1. The functions $G(z), F_{1}^{2}(z)$ and $F_{2}^{2}(z)$ are holomorphic in the open ball with radius 1 centered in $o$, that is, $o$ is not a singularity for any of these functions. Moreover, $z=1$ is a branch point for all of them and their only singularity in the complex plane.

Choice of the determination of the square root. We choose the determination of the square root with argument between $-\pi / 2$ and $\pi / 2$, that is, the function $h(w):=$ $\sqrt{|w|} \exp (i \arg (w) / 2)$, where $\arg (w)$ is chosen in the interval $[-\pi, \pi)$. That means that $\sqrt{1-z}$ is an holomorphic function defined in the open set $A:=\mathbb{C} \backslash\{z \in \mathbb{R}: z \geq 1\}$, and we extend it to $\{z \in \mathbb{R}: z \geq 1\}$ by continuity from the upper half plane (then we will not have continuity from the lower half plane). Note that this choice allows us to define $\sqrt{1-z+\sqrt{1-z}}$ as a holomorphic function in $A$ as well. 


\section{Estimates along the $y$-axis: the case $\xi \in[a, 1-c]$}

We first deal with the case of the asymptotic estimate for the transition probabilities $p^{(n)}\left(y_{k}, o\right)$, which we will rename for the sake of simplicity $p^{(n)}(k, 0)$. We first note that if $n$ and $k$ do not have the same parity, then $p^{(n)}(k, 0)=0$, while

$$
p^{(2 n+1)}(2 k+1,0)=\frac{1}{2}\left\{p^{(2 n)}(2 k+2,0)+p^{(2 n)}(2 k, 0)\right\} .
$$

Hence it will be enough to estimate $p^{(2 n)}(2 k, 0)$, which is given by the first equation of (3.4) (in this and in the following section we drop the index 1 from $F_{1}, \Psi_{\xi}^{1}$ and $\bar{\Psi}_{\xi}^{1}$ ).

LEMMA 4.1. The function $\Psi_{\xi}(z)$ has a unique minimum in $(0,1]$, namely $z_{o}(\xi)=$ $1-\xi^{2}$. Let $\phi(\xi)$ be this minimum, then $\phi(\xi)=\log \left((1-\xi)^{\xi-1}(1+\xi)^{-\xi-1}\right)$.

Here is the first estimate of our transition probabilities.

THEOREM 4.2. Let $a, c$ be positive numbers such that $a<1-c$. Then uniformly with respect to $\xi \in[a, 1-c]$,

$$
p^{(2 n)}(2 k, 0) \stackrel{n}{\sim} \frac{\sqrt{2 \xi}}{\sqrt{\pi(1+\xi)\left(1-\xi^{2}\right)}} e^{n \phi(\xi)} n^{-1 / 2} .
$$

PROOF. We first choose the curve of integration and split the integral into two parts (Part I of the proof); then we evaluate the part which will prove to be asymptotically negligible as compared to the other (Part II of the proof) and finally we estimate the main part (Part III of the proof).

Part I. The curve of integration is the circle with radius $z_{o}(\xi)$, centered in the origin

$$
\gamma: z(\xi, t)=z_{o}(\xi) e^{i t}, \quad t \in[-\pi, \pi] .
$$

We note that since $\xi \in[a, 1-c], z_{o}(\xi) \in[\bar{a}, 1-\bar{c}]$ for some $\bar{a}, \bar{c}>0$. Thus

$$
p^{(2 n)}(2 k, 0)=\frac{1}{2 \pi} \int_{-\pi}^{\pi} G(z(\xi, t)) \exp \left\{n \bar{\Psi}_{\xi}(t)\right\} d t .
$$

Now we want to write the Taylor expansion of $\bar{\Psi}_{\xi}(t)$ with Lagrange remainder, centered in $t=0$. This is possible since the third order derivative of $\bar{\Psi}_{\xi}(t)$ exists and is continuous in $t$, for all $\xi \in[a, 1-c]$. Hence we can write

$$
\bar{\Psi}_{\xi}(t)=\phi(\xi)-\left(1-\xi^{2}\right) t^{2} / 4 \xi^{2}+R(\xi, t),
$$

where $-\left(1-\xi^{2}\right) / 2 \xi^{2}=\bar{\Psi}_{\xi}^{\prime \prime}(0)$, the remainder is $R(\xi, t)=\bar{\Psi}_{\xi}^{\prime \prime \prime}(\bar{t}) t^{3} / 3$ !, and $\bar{t}$ is a point lying in the segment between 0 and $t$. 
We note that $\left|\bar{\Psi}_{\xi}^{\prime \prime \prime}(0)\right|>\varepsilon$ for all $\xi \in[a, 1-c]$ and for some $\varepsilon>0$. Since $\bar{\Psi}_{\xi}^{\prime \prime}(0)<0$, we can choose $\alpha>0$ such that $|R(\xi, t)| \leq-\bar{\Psi}_{\xi}^{\prime \prime}(0) t^{2} / 4$ for all $t \in[-\alpha, \alpha]$.

Now we split the integral (4.2) into two parts

$$
\begin{aligned}
& \text { A }:=\frac{1}{2 \pi} \int_{-\alpha}^{\alpha} G(z(\xi, t)) \exp \left\{n \bar{\Psi}_{\xi}(t)\right\} d t \\
& \text { B }:=\frac{1}{2 \pi} \int_{\alpha<|t| \leq \pi} G(z(\xi, t)) \exp \left\{n \bar{\Psi}_{\xi}(t)\right\} d t .
\end{aligned}
$$

Part II. By the definitions of $\bar{\Psi}_{\xi}, \phi$, and $z_{o}(\xi)$, we can write

$$
\mathbf{B}=\frac{e^{n \phi(\xi)}}{2 \pi} \int_{\alpha<|t| \leq \pi} G\left(z_{o}(\xi) e^{i t}\right)\left(\frac{F\left(z_{o}(\xi) e^{i t}\right)}{F\left(z_{o}(\xi)\right)}\right)^{2 n \xi} e^{-i n t} d t .
$$

The function $|F(z)| / F(|z|)$ is continuous in the compact set $K:=\{z \in \mathbb{C}:|z| \in$ $[\bar{a}, 1-\bar{c}], \alpha \leq \arg (z) \leq \pi\}$. By Lemma $2.5, \max _{z \in K}|F(z)| / F(|z|)=\lambda<1$. Moreover, again by Lemma $2.5,\left|G\left(z_{o}(\xi) e^{i t}\right)\right| \leq G(1-\bar{c})$, whence

$$
|\mathbf{B}| \leq e^{n \phi(\xi)} G(1-\bar{c}) \lambda^{2 a n},
$$

and this estimate is uniform with respect to $\xi \in[a, 1-c]$.

Part III. Expand $\bar{\Psi}_{\xi}(t)$ in the expression of $\mathbf{A}$ and perform the change of variable $\theta:=\sqrt{n} b(\xi) t$, where $b(\xi)=\sqrt{\left(1-\xi^{2}\right) / 2 \xi^{2}}$ (this change stresses the main term of the exponential)

$$
\mathbf{A}=\frac{e^{n \phi(\xi)}}{2 \pi b(\xi)} G\left(z_{o}(\xi)\right) n^{-1 / 2} \int_{-\alpha \sqrt{n} b(\xi)}^{\alpha \sqrt{n} b(\xi)} e^{-\theta^{2} / 2+n R\left(\xi, t_{n}\right)}\left(\frac{G\left(z_{o}(\xi) e^{i t_{n}}\right)}{G\left(z_{o}(\xi)\right)}\right) d \theta
$$

where $t_{n}:=\theta /(\sqrt{n} b(\xi))$. We want to give a uniform upper bound for the modulus of the integrand in order to apply Theorem 2.3 . Since by our choice of $\alpha,\left|n R\left(\xi, t_{n}\right)\right| \leq$ $\theta^{2} / 4$, the modulus of the integrand is bounded by $\exp \left\{-\theta^{2} / 4\right\}$ for all $n$ and $\xi \in$ $[a, 1-c]$. The integrand converges pointwise to $\exp \left\{-\theta^{2} / 2\right\}$, and the interval of integration converges to $\mathbb{R}$. Applying Theorem 2.3

$$
\mathbf{A} \stackrel{n}{\sim} \frac{e^{n \phi(\xi)}}{\sqrt{\pi}} n^{-1 / 2} \sqrt{\frac{2 \xi}{\left(1-\xi^{2}\right)(1+\xi)}}
$$

uniformly with respect to $\xi \in[a, 1-c]$. Finally, using (4.3) it is clear that $|\mathbf{B} / \mathbf{A}|$ tends to 0 when $n$ tends to infinity, uniformly with respect to $\xi \in[a, 1-c]$. 


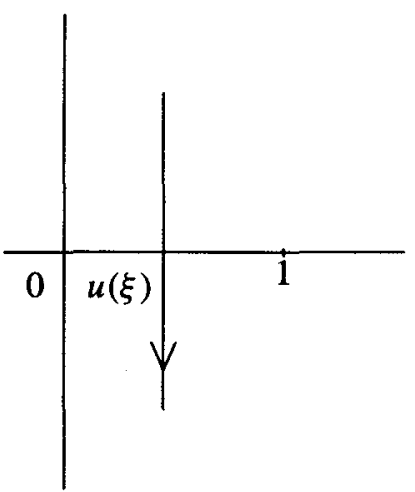

FIGURE 2. The segment in the $u$-plane.

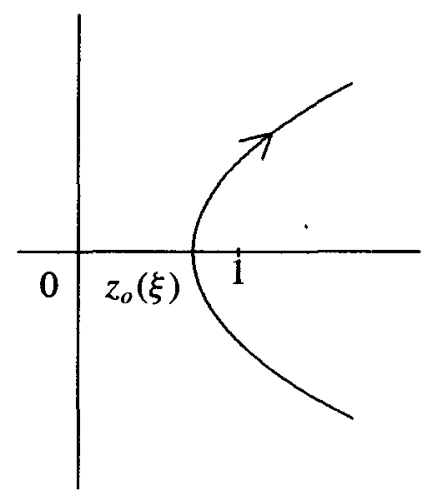

FIGURE 3. The curve in the $z$-plane.

\section{Estimates along the $y$-axis: the case $\xi \in[0, a]$}

If $\xi$ is allowed to tend to zero, the preceding estimate is no longer true. Then we have to choose a different curve of integration. We perform the change of variable $z=1-u^{2}$.

We note that, by our choice of the determination of the square root, if $\arg (u) \in$ $[-\pi / 2, \pi / 2)$ then $u=\sqrt{1-z}$ (the expression of $u$ in the other half plane will not be needed). The desired curve in the $u$-plane is simply a vertical segment whose parametrization is $u(\xi, t)=u(\xi)-i t$ where $u(\xi):=\sqrt{1-z_{o}(\xi)}=\xi$ and $t$ ranges from $-\alpha$ to $\alpha$ ( $\alpha$ will be chosen in the sequel). The segment is oriented downwards in order to produce a correctly oriented curve in the $z$-plane (see Figures 2-3).

The curve of integration in the $z$-plane will be the union of $\gamma_{1}: z(\xi, t)=1-u(\xi, t)^{2}$ for $|t| \leq \alpha$ and $\gamma_{2}: \tilde{z}(\xi, s):=|z(\xi, \alpha)| e^{i s}$ for $\arg (z(\xi, \alpha)) \leq s \leq 2 \pi-\arg (z(\xi, \alpha))$. Hence

$$
p^{(2 n)}(2 k, 0)=\mathbf{A}+\mathbf{B}=\frac{1}{2 \pi i} \int_{\gamma_{1}} \frac{G(z) F(z)^{2 k}}{z^{n+1}} d z+\frac{1}{2 \pi i} \int_{\gamma_{2}} \frac{G(z) F(z)^{2 k}}{z^{n+1}} d z
$$

Figure 4 shows how the contour of integration appears in the $z$-plane (the circle is elliptic due to a different choice of measure units on the horizontal and vertical axesindeed the integral on $\gamma_{2}$ is equal to that on any convex curve surrounding $o$ with the same endpoints).

The integral still makes sense, since, as we observed in Section 3, our Green functions can be extended to holomorphic functions defined in $\mathbb{C} \backslash\{z \in \mathbb{R}: z \geq 1\}$.

First we choose $a$ depending on $\alpha$ such that for all $\xi \in[0, a]$ we have $|z(\xi, \alpha)| \geq$ $1+\varepsilon_{o}$ for some fixed $\varepsilon_{o}>0$. This choice is possible since the mapping $\xi \mapsto z(\xi, \alpha)$ is continuous and $z(\xi, \alpha) \stackrel{\xi \rightarrow 0}{\longrightarrow} 1+\alpha^{2}$. 


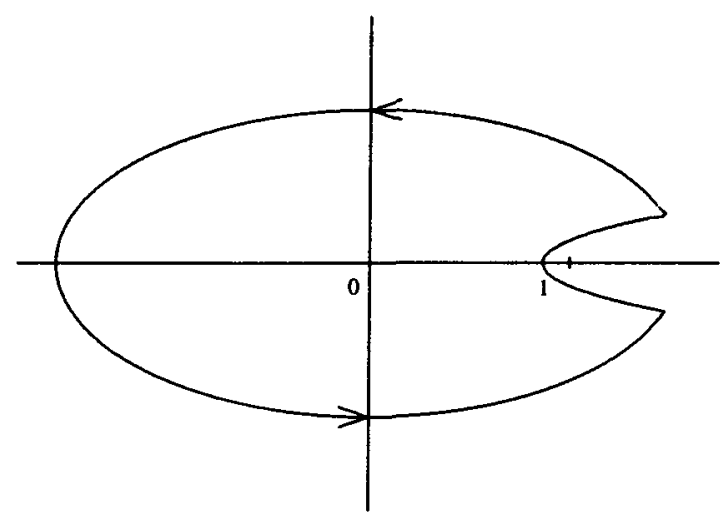

FIGURE 4. The curve of integration in the $z$-plane.

We observe that in this section we will make further choices of $a$, namely $a$ will be chosen sufficiently small in order to satisfy all the conditions we will find to be necessary. In the sequel we will not stress that when a new condition is introduced, if necessary $a$ is chosen smaller than before.

Now we estimate $\mathbf{B}$.

LEMMA 5.1. There exists $a>0$ such that

$$
\left|\frac{1}{2 \pi i} \int_{\gamma_{2}} \frac{G(z) F(z)^{2 k}}{z^{n+1}} d z\right| \leq C e^{n \phi(\xi)} \lambda^{n}
$$

for some $C>0, \lambda<1$ and for all $\xi \in[0, a]$.

Proof. Note that $\mathbf{B}$ can be written as

$$
\frac{e^{n \phi(\xi)}}{2 \pi} \int_{|t| \geq \arg (z(\xi, \alpha))} \frac{\left(F\left(|z(\xi, \alpha)| e^{i t}\right)\right)^{2 n \xi}}{F\left(z_{o}(\xi)\right)^{2 n \xi}} \frac{z_{o}(\xi)^{n}}{|z(\xi, \alpha)|^{n}} \frac{G\left(|z(\xi, \alpha)| e^{i t}\right)}{e^{i t n}} d t .
$$

We want to give an upper bound for $|G|$ and $\left|F^{2}\right|$, for all $\xi \in[0, a]$ and $|t| \geq$ $\arg (z(\xi, \alpha)$ ) (this upper bound will depend on $a$ ). This is possible since $K:=$ $\left\{z=|z(\xi, \alpha)| e^{i t}: \xi \in[0, a],|t| \geq \arg (z(\xi, \alpha))\right\}$ is a compact subset of $\mathbb{C}$. Since $d(K, 1) \geq \varepsilon_{o}$, there exists $C \geq 1$ (depending on $a$ ) such that

$$
\max _{z \in K}\left(\left|F^{2}(z)\right|,|G(z)|\right) \leq C .
$$

Hence the modulus of the integrand in (5.2) is bounded by

$$
\left(\frac{C}{F\left(z_{o}(a)\right)^{2}}\right)^{n a}\left(\frac{1}{1+\varepsilon_{o}}\right)^{n} C \text {. }
$$




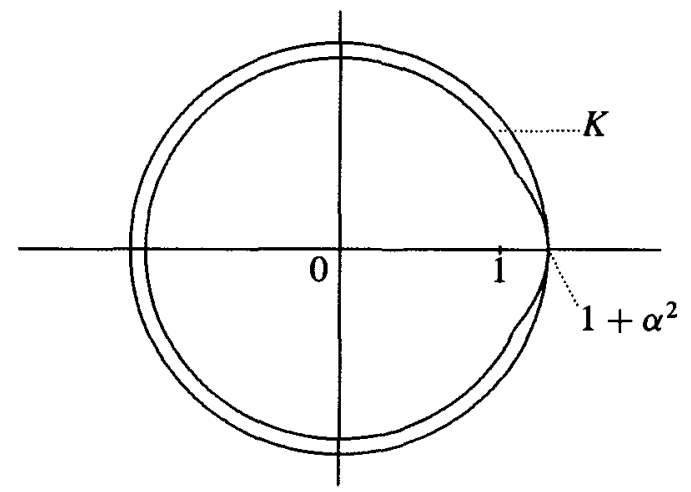

FIGURE 5. The compact set $K$.

We observe that if we take $C=C(a)$ to be the smallest possible $C \geq 1$ satisfying (5.3), then $C(a)$ turns out to be a continuous increasing function. Since $\left(C(a) / F\left(z_{0}(a)\right)^{2}\right)^{a} \rightarrow 1$ as $a \rightarrow 0$, then

$$
\lambda:=\left(\frac{C(a)}{F\left(z_{0}(a)\right)^{2}}\right)^{a}\left(\frac{1}{1+\varepsilon_{o}}\right)<1
$$

which leads to the conclusion.

Recalling that $z(\xi, t)=1-(\xi-i t)^{2}$, we have to estimate

$$
\mathrm{A}=\frac{1}{\pi} \int_{-\alpha}^{\alpha} \exp \left\{n \bar{\Psi}_{\xi}(t)\right\} \frac{G(z(\xi, t))}{z(\xi, t)}(\xi-i t) d t .
$$

LEMMA 5.2. The function $\bar{\Psi}_{\xi}(t)$ has a Taylor expansion centered in 0

$$
\bar{\Psi}_{\xi}(t)=\phi(\xi)-\frac{1}{2}\left(\frac{2}{1-\xi^{2}}\right) t^{2}+R(\xi, t)
$$

where $|R(\xi, t)| \leq C|t|^{3}$ for all $\xi \in[0, a],|t| \leq \alpha$, and for some $C>0$ (C depends on $a$ and $\alpha$ ).

Proof. We calculate the first derivative of $\bar{\Psi}_{\xi}$. Its Taylor series will lead us to the Taylor expansion of the primitive function:

$$
\bar{\Psi}_{\xi}^{\prime}(t)=-i\left\{\frac{1}{1+i t /(1-\xi)}-\frac{1}{1-i t /(1+\xi)}\right\}=\sum_{n \geq 1}\left[\frac{(-i)^{n+1}}{(1-\xi)^{n}}+\frac{(i)^{n+1}}{(1+\xi)^{n}}\right] t^{n},
$$


where the series converges to the function itself, provided that $|t /(1 \pm \xi)|<1$ (but this is true for $a$ and $\alpha$ sufficiently small). Then, since $\phi(\xi)=\bar{\Psi}_{\xi}(0)$,

$$
\bar{\Psi}_{\xi}(t)=\phi(\xi)-\frac{1}{1-\xi^{2}} t^{2}+\frac{4 i}{3} \frac{\xi}{\left(1-\xi^{2}\right)^{2}} t^{3}+t^{4} S(\xi, t),
$$

where $S(\xi, t)=\sum_{n \geq 4} n^{-1}\left[(-i)^{n} /(1-\xi)^{n-1}+i^{n} /(1+\xi)^{n-1}\right] t^{n-4}$. We want to show that $|S(\xi, t)| \leq C$ where $C>0$ does not depend on $\xi \in[0, a]$ nor on $t \in[-\alpha, \alpha]$. But

$$
|S(\xi, t)| \leq \sum_{n=4}^{\infty} \frac{1}{n}\left[\frac{1}{(1-a)^{n-1}}+1\right]|t|^{n-4} \leq \sum_{n=0}^{\infty}\left(\frac{2-a}{1-a}\right)^{n+3} \frac{\alpha^{n}}{n+4} .
$$

It is easy to see that this power series converges if $a \leq 1 / 2$ and $\alpha \leq 1 / 4$.

The asymptotic estimate turns out to be different depending on whether $\xi$ is allowed to tend very fast to 0 or not, that is, we have to distinguish two subcases.

THEOREM 5.3. For $a>0$ sufficiently small, uniformly with respect to $\xi \in\left[n^{-1 / 4}, a\right]$,

$$
p^{(2 n)}(2 k, 0) \stackrel{n}{\sim} \frac{\sqrt{2 \xi}}{\sqrt{\pi(1+\xi)\left(1-\xi^{2}\right)}} e^{n \phi(\xi)} n^{-1 / 2} .
$$

ProOF. We rewrite $\mathbf{A}$ using the Taylor expansion of $\bar{\Psi}_{\xi}(t)$ :

$$
\mathbf{A}=\frac{e^{n \phi(\xi)}}{\pi} \int_{-\alpha}^{\alpha} \frac{G(z(\xi, t))}{z(\xi, t)} \exp \left\{n\left[-\frac{1}{2}\left(\frac{2}{1-\xi^{2}}\right) t^{2}+R(\xi, t)\right]\right\}(\xi-i t) d t
$$

Let $b(\xi)=\sqrt{2 /\left(1-\xi^{2}\right)}$, and note that $b(\xi) \geq \sqrt{2}$. As in the case $\xi>a$, it is possible to choose $\alpha$ such that

$$
|R(\xi, t)| \leq \frac{1}{4} b(\xi)^{2} t^{2}, \quad \forall|t| \leq \alpha, \forall \xi \in[0, a] .
$$

Perform a change of variable in order to stress the main term of the exponential $\theta:=\sqrt{n} b(\xi) t$. If we put $t_{n}=\theta /(\sqrt{n} b(\xi)), \alpha_{n}=\alpha \sqrt{n} b(\xi)$ and $z_{n}=z\left(\xi, t_{n}\right)$,

$$
\mathbf{A}=\frac{e^{n \phi(\xi)}}{\pi \sqrt{n} b(\xi)} \frac{G\left(z_{o}(\xi)\right)}{z_{o}(\xi)} \int_{-\alpha_{n}}^{\alpha_{n}} \frac{G\left(z_{n}\right)}{G\left(z_{o}(\xi)\right)} \frac{z_{o}(\xi)}{z_{n}} e^{-\theta^{2} / 2+n R\left(\xi, t_{n}\right)}\left(\xi-i t_{n}\right) d \theta
$$

We want to give an upper bound (valid for all $\xi \in\left[n^{-1 / 4}, a\right]$ ) for the modulus of the integrand in order to apply Theorem 2.3. The exponential part is bounded by $e^{-\theta^{2} / 4}$ 
and $\xi-i t_{n} \stackrel{n}{\sim} \xi$ uniformly with respect to $\xi \in\left[n^{-1 / 4}, a\right]$, for every fixed $\theta$, as $n$ tends to infinity. For all $|t| \leq \alpha$ and $\xi \in\left[n^{-1 / 4}, a\right]$,

$$
\left|\frac{G\left(z_{n}\right)}{G\left(z_{o}(\xi)\right)}\right|\left|\frac{z_{0}(\xi)}{z_{n}}\right| \leq 1 .
$$

Then if in (5.6) we extract the factor $\xi$ from the integral, the modulus of the new integrand is bounded (for all $\xi \in\left[n^{-1 / 4}, a\right]$ ) by $e^{-\theta^{2} / 4}(1+c|\theta|$ ) and the integrand converges pointwise to $e^{-\theta^{2} / 2}$. Apply Theorem 2.3 to obtain

$$
\mathbf{A} \stackrel{n}{\sim} \frac{\sqrt{2 \xi}}{\sqrt{\pi(1+\xi)\left(1-\xi^{2}\right)}} e^{n \phi(\xi)} n^{-1 / 2}
$$

uniformly with respect to $\xi \in\left[n^{-1 / 4}, a\right]$. The theorem follows since, by Lemma 5.1 , $\left[\mathbf{B} / \mathbf{A} \mid\right.$ tends to zero as $n$ tends to infinity, uniformly with respect to $\xi \in\left[n^{-1 / 4}, a\right]$.

When $\xi \in\left[0, n^{-1 / 4}\right]$ it is no longer true that $\xi-i t_{n} \sim \xi$ and the technique will be slightly different. A useful tool will appear to be the computation of the real and imaginary parts of $\sqrt{\xi-i t_{n}}$.

LEMMA 5.4. Let $\sqrt{\xi-i t}=a(\xi, t)+i b(\xi, t)$. Then

$$
a(\xi, t)=\sqrt{\left(\sqrt{\xi^{2}+t^{2}}+\xi\right) / 2}, \quad b(\xi, t)=-\operatorname{sign}(t) \sqrt{\left(\sqrt{\xi^{2}+t^{2}}-\xi\right) / 2}
$$

and both these terms are $O(\sqrt{\xi})+O(\sqrt{|t|})$.

THEOREM 5.5. Uniformly with respect to $\xi \in\left[0, n^{-1 / 4}\right]$,

$$
p^{(2 n)}(2 k, 0) \stackrel{n}{\sim} \frac{I(\sqrt{n \xi})}{\sqrt{2} \pi} e^{n \phi(\xi)} n^{-3 / 4},
$$

where $I(t):=\int_{\mathbb{R}} e^{-\theta^{2} / 2} \sqrt{\sqrt{t^{2}+\theta^{2} / 2}+t} d \theta$. Moreover for all $\varepsilon>0$, uniformly with respect to $\xi \in\left[0, n^{-1 / 2-\varepsilon}\right]$,

$$
p^{(2 n)}(2 k, 0) \stackrel{n}{\sim} \frac{\sqrt{2} e^{n \phi(\xi)}}{\Gamma(1 / 4)} n^{-3 / 4} .
$$

Proof. The integral we have to estimate is still $\mathbf{A}$ of (5.4). Choose $\alpha$ as in Theorem 5.3 and let $\theta, b(\xi), t_{n}, \alpha_{n}$ and $z_{n}$ be as defined there. Then we proceed differently: in Part I we show a decomposition of $G(z)$ into its singular and regular parts; Part II is devoted to the estimate of the real part of the integrand in A: we stress only the terms which are not $o(\xi)$ or $o\left(t_{n}^{2}\right)$ (uniformly with respect to $\xi \in\left[0, n^{-1 / 4}\right]$ ). In Part III we write $\mathbf{A}=\mathbf{A}_{1}+\mathbf{A}_{2}+\mathbf{A}_{3}$. In Part IV we estimate $\mathbf{A}_{1}$ and describe some properties of $I(t)$. Part $V$ shows negligibility of $\mathbf{B}, \mathbf{A}_{2}$ and $\mathbf{A}_{3}$. 
Part I. We write a decomposition for $G(z)$ :

$$
G(z)=(1-z)^{-1 / 4} H(z)+(1-z)^{1 / 4} K(z),
$$

where $H(z):=\sqrt{2} \sum_{n=0}^{\infty}\left(\begin{array}{c}-1 / 2 \\ 2 n\end{array}\right)(1-z)^{n}$ and $K(z):=\sqrt{2} \sum_{n=0}^{\infty}\left(\begin{array}{c}-1 / 2 \\ 2 n+1\end{array}\right)(1-z)^{n}$ are two holomorphic functions defined in a disc centered in $z=1$ and with radius $r$ greater or equal to $2 / 3$. Note that we need $|1-z|<r$, but this surely holds in the integration domain if $\alpha$ is sufficiently small. Moreover, we decompose $H$ and $K$ into their real and imaginary parts: $H=H_{0}+i H_{1}$ and $K=K_{0}+i K_{1}$.

Part II. In A we decompose $G(z)$ as in (5.7), the remainder term $R$ in its real and imaginary parts $R_{0}$ and $R_{1}$ respectively:

$$
\mathbf{A}=\frac{e^{n \phi(\xi)}}{\pi \sqrt{n} b(\xi)} \int_{-\alpha_{n}}^{\alpha_{n}} e^{-\theta^{2} / 2+n R_{0}\left(\xi, t_{n}\right)+i n R_{1}\left(\xi, t_{n}\right)} \frac{a_{n}+i b_{n}}{z_{n}}\left(H\left(z_{n}\right)+\left(a_{n}+i b_{n}\right)^{2} K\left(z_{n}\right)\right) d \theta,
$$

where $a_{n}=a\left(\xi, t_{n}\right)$ and $b=b\left(\xi, t_{n}\right)$. Since the transition probabilities are non negative quantities, we are interested only in the real part of the last integral (in fact the imaginary part is 0 , but taking into account only the real part of the integrand avoids useless computation).

In order to apply Theorem 2.4, we estimate the function (depending on $\xi, n$ and $\theta$ ) to which the real part of our integrand is asymptotic. In the following estimates every $o$ and $O$ is understood to be uniform with respect to $\xi \in\left[0, n^{-1 / 4}\right]$.

First we estimate the main term of

$$
\operatorname{Re}\left\{e^{i n R_{1}\left(\xi, t_{n}\right)}\left(a_{n}+i b_{n}\right)\left(H\left(z_{n}\right)+\left(a_{n}+i b_{n}\right)^{2} K\left(z_{n}\right)\right)\right\} .
$$

Using the expression of $R_{1}$ which can be deduced from the proof of Lemma 5.2, one proves that $R_{1}(\xi, t)=t^{3}(O(\xi)+O(t))$, whence for every fixed $\theta$

$$
n R_{1}\left(\xi, t_{n}\right)=t_{n}\left(O(\xi)+O\left(t_{n}\right)\right) .
$$

Then (5.8) can be written as

$$
\cos \left(n R_{1}\left(\xi, t_{n}\right)\right) a_{n} H_{0}\left(z_{n}\right)+\cos \left(n R_{1}\left(\xi, t_{n}\right)\right) b_{n} t_{n} K_{0}\left(z_{n}\right)+o(\xi)+o\left(t_{n}^{2}\right),
$$

(the proof is tedious but straightforward). The only terms which cannot be immediately seen as either $o(\xi)$ or $o\left(t_{n}^{2}\right)$ are

$$
\xi O\left(\sqrt{\left|t_{n}\right|}\right)=o(\xi), \quad O(\xi) O\left(\left|t_{n}\right|^{3 / 2}\right)=o(\xi), \quad O(\sqrt{\xi}) O\left(t_{n}^{2}\right)=o\left(t_{n}^{2}\right),
$$

and these estimates are uniform with respect to $\xi \in\left[0, n^{-1 / 4}\right]$, since we consider $n$ tending to infinity, which implies that both $\xi$ and $t_{n}$ tend to 0 (for every fixed $\theta$ ).

Finally, noting that $z_{n}^{-1}=1+O\left(\xi^{2}\right)+O\left(t_{n}^{2}\right)$ for every fixed $\theta$,

$$
\begin{aligned}
\mathbf{A}= & \frac{e^{n \phi(\xi)}}{\pi \sqrt{n} b(\xi)} \int_{-\alpha_{n}}^{\alpha_{n}} e^{-\theta^{2} / 2+n R_{0}\left(\xi, t_{n}\right)}\left(1+O\left(\xi^{2}\right)+O\left(t_{n}^{2}\right)\right) \\
& \times\left\{\cos \left(n R_{1}\left(\xi, t_{n}\right)\right) a_{n} H_{0}\left(z_{n}\right)+\cos \left(n R_{1}\left(\xi, t_{n}\right)\right) b_{n} t_{n} K_{0}\left(z_{n}\right)+o(\xi)+o\left(t_{n}^{2}\right)\right\} d \theta .
\end{aligned}
$$


Part III. If in the preceding integral we write $a_{n}$ and $b_{n}$ as functions of $\theta, \xi$ and $n$ and we extract from the integral the terms which do not depend on $\theta$, we can split $\mathbf{A}$ in three parts

$$
\begin{aligned}
\mathbf{A}_{1}= & \frac{e^{n \phi(\xi)}\left(1-\xi^{2}\right)^{3 / 4}}{\sqrt{2} \pi n^{3 / 4}} \int_{-\alpha_{n}}^{\alpha_{n}} e^{-\theta^{2} / 2+n R_{0}\left(\xi, t_{n}\right)}\left(1+O\left(\xi^{2}\right)+O\left(t_{n}^{2}\right)\right) \\
& \times \cos \left(n R_{1}\left(\xi, t_{n}\right)\right) \sqrt{\sqrt{\frac{\theta^{2}}{2}+\frac{n \xi^{2}}{\left(1-\xi^{2}\right)}}+\frac{\sqrt{n \xi}}{\sqrt{1-\xi^{2}}}} \frac{H_{0}\left(z_{n}\right)}{\sqrt{2}} d \theta, \\
\mathbf{A}_{2}= & \frac{e^{n \phi(\xi)}\left(1-\xi^{2}\right)^{5 / 4}}{4 \pi n^{5 / 4}} \int_{-\alpha_{n}}^{\alpha_{n}} e^{-\theta^{2} / 2+n R_{0}\left(\xi, t_{n}\right)}\left(1+O\left(\xi^{2}\right)+O\left(t_{n}^{2}\right)\right) \\
& \times \cos \left(n R_{1}\left(\xi, t_{n}\right)\right) \frac{\theta^{2}}{\sqrt{\sqrt{\frac{\theta^{2}}{2}+\frac{n \xi^{2}}{\left(1-\xi^{2}\right)}}+\frac{\sqrt{n \xi}}{\sqrt{1-\xi^{2}}}}}\left(-K_{0}\left(z_{n}\right)\right) d \theta, \\
\mathbf{A}_{3}= & \frac{e^{n \phi(\xi)}\left(1-\xi^{2}\right)^{1 / 2}}{\sqrt{2} \pi n^{1 / 2}} \int_{-\alpha_{n}}^{\alpha_{n}} e^{-\theta^{2} / 2+n R_{0}\left(\xi, t_{n}\right)}\left(1+O\left(\xi^{2}\right)+O\left(t_{n}^{2}\right)\right)\left(o(\xi)+O\left(t_{n}^{2}\right)\right) d \theta .
\end{aligned}
$$

Part IV. We show that the integral in $\mathbf{A}_{1}$ is asymptotic to

$$
I(\sqrt{n} \xi)=\int_{\mathbb{R}} e^{-\theta^{2} / 2} \sqrt{\sqrt{n \xi^{2}+\theta^{2} / 2}+\sqrt{n} \xi} d \theta .
$$

We want to apply Theorem 2.4 to the integrand of $\mathbf{A}_{1}$, but a new problem raises: the quantity $\sqrt{n} \xi$ may tend to 0 or to $+\infty$ as well. Hence we introduce a new function, namely $Q(n, \xi):=\max \{1, \sqrt{n} \xi\}$. We rewrite $\mathbf{A}_{1}$

$$
\begin{aligned}
& \frac{e^{n \phi(\xi)}\left(1-\xi^{2}\right)^{3 / 4} \sqrt{Q(n, \xi)}}{\sqrt{2} \pi n^{3 / 4}} \int_{-\alpha_{n}}^{\alpha_{n}} e^{-\theta^{2} / 2+n R_{0}\left(\xi, t_{n}\right)}\left(1+O\left(\xi^{2}\right)+O\left(t_{n}^{2}\right)\right) \frac{H_{0}\left(z_{n}\right)}{\sqrt{2}} \\
& \times \cos \left(n R_{1}\left(\xi, t_{n}\right)\right) \sqrt{\sqrt{\frac{\theta^{2}}{2 Q^{2}(n, \xi)}+\frac{n \xi^{2}}{\left(1-\xi^{2}\right) Q^{2}(n, \xi)}}+\frac{\sqrt{n} \xi}{\sqrt{1-\xi^{2}} Q(n, \xi)}} d \theta .
\end{aligned}
$$

We evaluate the (uniform) asymptotic value of the integrand (which we call $f_{n}(\theta, \xi)$, defined for all $\theta \in \mathbb{R}$ ). Recalling that $\left|n R_{0}\left(\xi, t_{n}\right)\right| \rightarrow 0$ for every fixed $\theta$, uniformly with respect to $\xi \in\left[0, n^{-1 / 4}\right]$, one shows that $f_{n}(\theta, \xi)$ is asymptotic to

$$
h_{n}(\theta, \xi):=e^{-\theta^{2} / 2} \sqrt{\sqrt{\frac{\theta^{2}}{2 Q^{2}(n, \xi)}+\frac{n \xi^{2}}{\left(1-\xi^{2}\right) Q^{2}(n, \xi)}}+\frac{\sqrt{n} \xi}{\sqrt{1-\xi^{2}} Q(n, \xi)}} .
$$

Thus $f_{n}$ and $h_{n}$ are both bounded, for every $\xi \in\left[0, n^{-1 / 4}\right]$, by

$$
C e^{-\theta^{2} / 4} \sqrt{\sqrt{1+\theta^{2} / 2}+1}
$$


Finally, $\left|\int_{\mathbb{R}} h_{n}(\theta, \xi) d \theta\right|>c$ for some $c>0$, for all $n$ and $\xi \in\left[0, n^{-1 / 4}\right]$. By Theorem 2.4,

$$
\mathbf{A}_{1} \stackrel{n}{\sim} \frac{e^{n \phi(\xi)}}{\sqrt{2} \pi n^{3 / 4}} I(\sqrt{n \xi})
$$

Part V. By Lemma 5.1, $\left|\mathbf{B} / \mathbf{A}_{1}\right|$ tends to 0 uniformly with respect to $\xi \in\left[0, n^{-1 / 4}\right]$. Observing that $\sqrt{\sqrt{x^{2}+\theta^{2} / 2}+x} \geq 2^{-1 / 4}|\theta|^{1 / 2}$ for all $x \geq 0$, one obtains that

$$
\left|\mathbf{A}_{2}\right| \leq C e^{n \phi(\xi)} n^{-5 / 4} \int_{\mathbb{R}} e^{-\theta^{2} / 4}|\theta|^{3 / 2} d \theta
$$

whence the negligibility of $\mathbf{A}_{2}$ with respect to $\mathbf{A}_{1}$.

As for $\mathbf{A}_{3}$, one can prove that $\left|\mathbf{A}_{3}\right| \leq C e^{n \phi(\xi)} n^{-3 / 4}\left(\xi^{1 / 8}+n^{-1 / 4}\right)$, once he observes that every $o(\xi)$ besides those in (5.9) is also equal to $\xi^{1 / 8} o(\xi)$, that $\left|o\left(t_{n}^{2}\right)\right| \leq C \theta / n$, and that for the $o(\xi)$ in (5.9)

$$
\begin{gathered}
\frac{\xi O\left(\sqrt{\left|t_{n}\right|}\right)}{\xi} \leq C \sqrt{\left|t_{n}\right|} \leq C \theta^{1 / 2} n^{-1 / 4}, \\
\frac{O(\xi) O\left(\left|t_{n}\right|^{3 / 2}\right)}{\xi} \leq C\left|t_{n}\right|^{3 / 2} \leq C \theta^{3 / 2} n^{-3 / 4} .
\end{gathered}
$$

The proof of the first estimate is now complete, the statement for $\xi \in\left[0, n^{-1 / 2-\varepsilon}\right]$ is proved in the same way, once we note that $I(t)$ is continuous in $0, I(0)=\sqrt{2} \Gamma(3 / 4)$, and $\Gamma(3 / 4)=(\sqrt{2} \pi) / \Gamma(1 / 4)$. Note that the same holds under the weaker assumption that $\sqrt{n} \xi \rightarrow 0$ uniformly in $\xi$ and that the estimate agrees with Theorem 5.3 for $\xi=n^{-1 / 4}$ since $I(t) \sim 2 \sqrt{t \pi}$ as $t \rightarrow \infty$.

\section{Estimates along the $x$-axis: the case $\xi \in[a, 1-c]$}

In this section we give an asymptotic estimate for the transition probabilities $p^{(n)}\left(x_{k}, o\right)$, which we will rename $p^{(n)}(k, 0)$. We first note that if $n$ and $k$ do not have the same parity, then $p^{(n)}(k, 0)=0$, but we cannot repeat the trick we used on the $y$-axis to derive $p^{(2 n+1)}(2 k+1,0)$ from $p^{(2 n)}(2 k, 0)$. We estimate only the second type of transition probabilities (the first ones can be derived in a similar way-see Section 10).

The basic idea underlying our proofs here is essentially the same as for the case of the $y$-axis, nevertheless much more technical difficulties arise and the techniques we employ appear more involved. We drop the index 2 from $F_{2}, \Psi_{\xi}^{2}$ and $\bar{\Psi}_{\xi}^{2}$, so here we use the same symbols (among which also $\phi$ ) for functions and values which are not the same but play the same role as the analogues for the $y$-axis. 
LEMMA 6.1. The function $\Psi_{\xi}(z)$ has a unique minimum (which we call $\phi(\xi)$ ) in $(0,1]$, namely $z_{o}(\xi)=1-u_{o}(\xi)^{2}$, where $u_{o}(\xi)=\xi^{2 / 3}\left[54+6 \sqrt{81-6 \xi^{2}}\right]^{1 / 3} / 6+$ $\xi^{4 / 3}\left[54+6 \sqrt{81-6 \xi^{2}}\right]^{-1 / 3}$.

The following theorem is the analogue of Theorem 4.2 for the $x$-axis (and the proof is just the same).

THEOREM 6.2. Let $a, c$ be positive numbers such that $a<1-c$. Then uniformly with respect to $\xi \in[a, 1-c]$,

$$
p^{(2 n)}(2 k, 0) \stackrel{n}{\sim} \frac{\sqrt{2\left(1-z_{o}(\xi)\right)} G\left(z_{o}(\xi)\right)}{\sqrt{\pi\left(1+2 z_{o}(\xi)-\sqrt{1-z_{o}(\xi)}\right)}} e^{n \phi(\xi)} n^{-1 / 2} .
$$

\section{Estimates along the $x$-axis: the case $\xi \in[0, a]$}

We proceed as in Section 5, with the change of variable $z=1-v^{4}$ instead of $z=1-u^{2}$. The integral we are going to estimate has the same expression of (5.1) ( $F$ of course here is $F_{2}$ ) where $\gamma_{1}: z(\xi, t)=1-v(\xi, t)^{4}$ for $|t| \leq \alpha$ (for any fixed $\xi, v(\xi, t)$ is a suitable curve in the $v$-plane) and $\gamma_{2}: \tilde{z}(\xi, s)=|z(\xi, \alpha)| e^{i s}$ for $\arg (z(\xi, \alpha)) \leq s \leq 2 \pi-\arg (z(\xi, \alpha))$.

The desired curve in the $v$-plane is a line whose parametrization is

$$
v(\xi, t)= \begin{cases}v(\xi)+e^{i \beta} t & \text { if } t \in[0, \alpha] \\ v(\xi)-e^{-i \beta} t & \text { if } t \in[-\alpha, 0),\end{cases}
$$

where $v(\xi):=\sqrt{u_{o}(\xi)}\left(u_{o}(\xi)\right.$ is defined in Lemma 6.1), and $\alpha$ and $\beta$ will be chosen in the sequel.

If we look at the proofs of Theorem 5.3 and Theorem 5.5 we see that, once the integration contour is fixed to be $\gamma_{1} \cup \gamma_{2}$ and integration on $\gamma_{2}$ is proved to be negligible, the first steps of the procedure are (roughly speaking):

(1) to write a Taylor expansion of $\bar{\Psi}_{\xi}(t):=\Psi_{\xi}(z(\xi, t))$ for $|t| \leq \alpha$;

(2) to find a change of variable $\theta=f(n, \xi, t)$ such that $n$ 'disappears' from one term of the exponential in the integral and the remaining terms of the expansion are negligible.

Our first task is to prove that $\bar{\Psi}_{\xi}(t)$ has a Taylor series expansion and to estimate its first terms. This is a matter of quite long computations that we omit here, we simply exhibit the results.

In case that the reader wants to perform these computations, we point out that one zan write first the series in $v$ of $\Psi_{\xi}\left(1-v^{4}\right)$ and then substitute $v=v(\xi, t)$ to obtain the series in $t$. In doing so, particular attention must be paid in computing the roots (for 
instance $\sqrt{v^{4}}$ is not necessarily equal to $v^{2}$ ) whose explicit expressions depend on the position of $v$ in the complex plane, with respect to the two bisectors of the quadrants.

LEMMA 7.1. The function $\bar{\Psi}_{\xi}(t)$ has a Taylor series expansion centered in 0 , with positive radius of convergence not depending on $\xi$, and the following equality holds:

$$
\bar{\Psi}_{\xi}(t)=\phi(\xi)+e^{2 i \beta} a_{2}(\xi) t^{2}+e^{3 i \beta} a_{3}(\xi) t^{3}+e^{4 i \beta} a_{4}(\xi) t^{4}+R(\xi, t)
$$

where the remainder term is $R(\xi, t)=O\left(t^{5}\right)$, uniformly with respect to $\xi \in[0, a]$. Moreover, if $\xi \rightarrow 0$,

$$
a_{2}(\xi) \sim 3 \cdot 2^{2 / 3} \xi^{2 / 3} ; \quad a_{3}(\xi) \sim 2^{11 / 6} \xi^{1 / 3} ; \quad a_{4}(\xi) \sim 1 .
$$

Now we have to choose the curve of integration (that is, $\beta$ ) in order to obtain that

(a) the curve $z(\xi, t)=1-v(\xi, t)^{4}$ has some 'good properties' (for instance there exists $\alpha>0$ such that $|z(\xi, \alpha)| \geq 1+\varepsilon_{0}$ for some $\varepsilon_{0}>0$ and for every $\xi$ in the considered range);

(b) we can dominate $\exp \left\{n \bar{\Psi}_{\xi}(t)\right\}$ with an integrable function.

We observe that, as for the integral on $\gamma_{1}$, we can consider only the piece of $\gamma_{1}$ lying in the first quadrant (that is the one corresponding to $t \geq 0$ ). Indeed, since $\gamma_{1}$ is symmetric with respect to the horizontal axis, it is easy to see that

$$
\mathbf{A}=\frac{1}{\pi} \operatorname{Im} \int_{0}^{\alpha} \frac{G(z(\xi, t))}{z(\xi, t)} e^{n \bar{\psi}_{\xi}(t)}\left[-4 e^{i \beta}\left(v(\xi)+e^{i \beta} t\right)^{3}\right] d t .
$$

Requirement (b) restricts the range of $\beta$. In fact we require that the real parts of the expansion in Lemma 7.1 have non positive coefficients, that is, that $\cos (2 \beta), \cos (3 \beta)$ and $\cos (4 \beta)$ are all non positive. This corresponds to $\beta \in \Delta:=[\pi / 4,3 \pi / 8] \cup$ $[-3 \pi / 8,-\pi / 4]$.

REMARK 7.2. Among the curves of the family $\mathscr{F}:=\{z(\xi, t): t \geq 0, \beta \in \Delta\}$, the ones with $\beta= \pm \pi / 4$ have exactly one intersection with the real axis: $z(\xi, 0)=$ $1-v(\xi)^{4}$; while the others have exactly two intersections with the same axis: one for $t=0$ and the other for $t=v(\xi) /(\sin \beta-\cos \beta)$ if $\beta \in(\pi / 4,3 \pi / 8]$ or for $t=-v(\xi) /(\sin \beta+\cos \beta)$ if $\beta \in[-3 \pi / 8,-\pi / 4]$.

We present the plot of some of the curves we will use. Since the curve for $\beta=\pi / 4$ turns clockwise (with respect to the origin), we will prefer its conjugate, that is, the curve for $\beta=-\pi / 4$ (see Figure 6 ).

Figure 7 shows the curve for $\beta=\pi / 3$, where we used a logarithmic scale on the horizontal axis in order to show the 'pathological' behaviour of the curves of the family with $\beta \neq \pm \pi / 4$. 


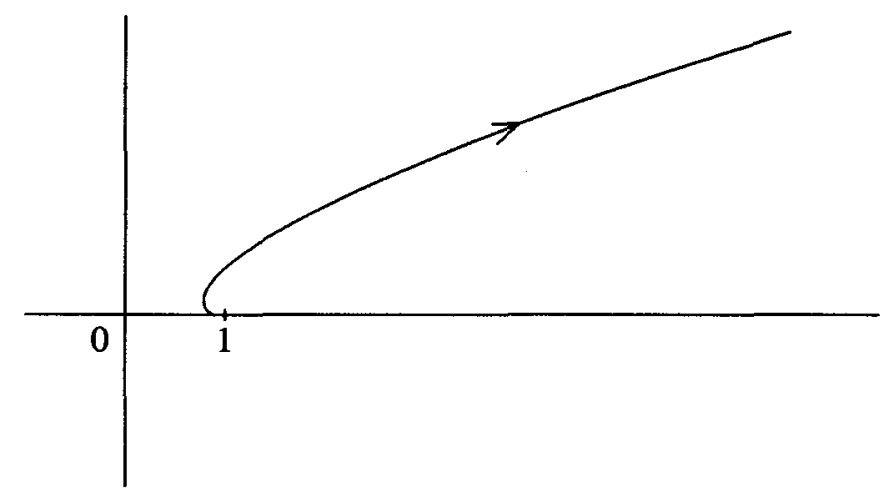

FIGURE 6. The curve $\gamma_{1 \mid t \geq 0}$ for $\beta=-\pi / 4$.

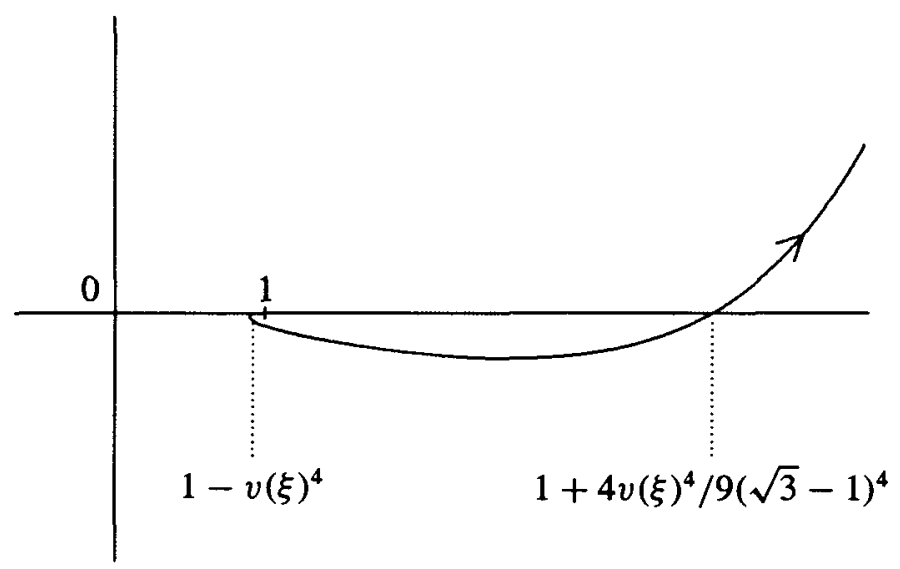

FIGURE 7. The curve $\gamma_{1_{\mid t \geq 0}}$ for $\beta=\pi / 3$.

The choice of a proper change of variable is between three substitutions, each stressing a different piece of the exponential part of the integrand:

(a) $n a_{2}(\xi) t^{2}=\theta^{2}$, or (b) $n a_{3}(\xi) t^{3}=\theta^{3}$, or (c) $n a_{4}(\xi) t^{4}=\theta^{4}$.

LEMMA 7.3. In order to have an upper bound for $n \bar{\Psi}_{\xi}(t(\theta))$, we must use substitution (a) for $\xi \geq n^{-3 / 4}$, substitution (c) for $\xi \leq n^{-3 / 4}$.

ProOF. With substitution (a), $e^{n \bar{\Psi}_{\xi}(t)}$ can be written as

$$
e^{n \phi(\xi)} \exp \left\{e^{2 i \beta} \theta^{2}+e^{3 i \beta} \frac{a_{3}(\xi)}{\sqrt{n}\left(a_{2}(\xi)\right)^{3 / 2}} \theta^{3}+e^{4 i \beta} \frac{a_{4}(\xi)}{n\left(a_{2}(\xi)\right)^{2}} \theta^{4}+n R\left(\xi, t_{n}\right)\right\}
$$


Then for $a$ sufficiently small and for every $\xi \in[0, a]$,

$$
a_{3}(\xi) / \sqrt{n}\left(a_{2}(\xi)\right)^{3 / 2} \leq C / n^{1 / 2} \xi^{2 / 3}, \quad a_{4}(\xi) / n\left(a_{2}(\xi)\right)^{2} \leq C / n \xi^{4 / 3},
$$

which are all surely bounded if $\xi \geq n^{-3 / 4}$.

Similarly, with substitution (c), the coefficients of $\theta^{2}$ and $\theta^{3}$ are bounded for $\xi \leq n^{-3 / 4}$, while the coefficient of $\theta^{4}$ is $e^{4 i \beta}$.

Finally, with substitution (b) the coefficient of $\theta^{2}$ is bounded if $\xi \leq n^{-3 / 4}$, while the coefficient of $\theta^{4}$ is bounded if $\xi \geq n^{-3 / 4}$. This makes substitution (b) a not suitable one.

\section{Estimates along the $x$-axis: the case $\xi \in\left[0, n^{-3 / 4}\right]$}

In this section we fix $\beta=-\pi / 4$ and $z(\xi, t)=1-\left(v(\xi)+e^{-i \pi / 4} t\right)^{4}$. The curve of integration is similar to that in Figure 4, even if $\gamma_{1}$ is here the arc of the curve in Figure 6 corresponding to $t \in[0, \alpha]$, plus the arc obtained by symmetry with respect to the horizontal axis.

We choose $a$ depending on $\alpha$ such that for all $\xi \in[0, a]$ we have $|z(\xi, \alpha)| \geq 1+\varepsilon_{o}$ for some fixed $\varepsilon_{o}>0$. Thanks to this choice, the circular part of the curve of integration will be far from the singularity $z=1$. This choice is possible since the mapping $\xi \mapsto z(\xi, \alpha)$ is continuous and $z(\xi, \alpha) \stackrel{\xi \rightarrow 0}{\longrightarrow} 1+\alpha^{4}$.

The proof of the following lemma is analogous to that of Lemma 5.1.

LEMMA 8.1. There exists $a>0$ such that

$$
\left|\frac{1}{2 \pi i} \int_{\gamma_{2}} \frac{G(z) F(z)^{2 k}}{z^{n+1}} d s\right| \leq C e^{n \phi(\xi)} \lambda^{n}
$$

for some $C>0, \lambda<1$ and for all $\xi \in[0, a]$.

THEOREM 8.2. Uniformly with respect to $\xi \in\left[0, n^{-3 / 4}\right]$,

where

$$
p^{(2 n)}(2 k, 0) \stackrel{n}{\sim} \frac{4}{\pi} I\left(n^{1 / 4} \xi^{1 / 3}\right) e^{n \phi(\xi)} n^{-3 / 4},
$$

$$
\begin{aligned}
I(t):= & \int_{0}^{+\infty} e^{-2^{4 / 3} \theta^{3}-\theta^{4}}\left[\cos \left(3 \cdot 2^{2 / 3} t^{2} \theta^{2}-2^{4 / 3} t \theta^{3}\right)\left(2^{-1 / 3} t^{2}+\theta^{2}+2^{4 / 3} t \theta\right)\right. \\
& \left.+\sin \left(3 \cdot 2^{2 / 3} t^{2} \theta^{2}-2^{4 / 3} t \theta^{3}\right)\left(\theta^{2}-2^{-1 / 3} t^{2}\right)\right] d \theta .
\end{aligned}
$$

Moreover for all $\varepsilon>0$, uniformly with respect to $\xi \in\left[0, n^{-3 / 4-\varepsilon}\right]$,

$$
p^{(2 n)}(2 k, 0) \stackrel{n}{\sim} \frac{\sqrt{2}}{\Gamma(1 / 4)} e^{n \phi(\xi)} n^{-3 / 4}
$$


Proof. Perform the change of variable (c), put $t_{n}=\theta / \sqrt[4]{n a_{4}(\xi)}, z_{n}=z\left(\xi, t_{n}\right)$ and $\alpha_{n}=\sqrt[4]{n a_{4}(\xi)} \alpha$. Then

$$
\begin{aligned}
\mathbf{A}= & \frac{-2 \sqrt{2} e^{n \phi(\xi)}}{\pi \sqrt[4]{n a_{4}(\xi)}}(\operatorname{Im}-\operatorname{Re}) \int_{0}^{\alpha_{n}} \frac{G\left(z_{n}\right)}{z_{n}}\left(v(\xi)+e^{-i \pi / 4} t_{n}\right)^{3} \\
& \times \exp \left\{-i \frac{n^{1 / 2} a_{2}(\xi)}{a_{4}(\xi)^{1 / 2}} \theta^{2}-(1+i) \frac{n^{1 / 4} a_{3}(\xi)}{\sqrt{2} a_{4}(\xi)^{3 / 4}} \theta^{3}-\theta^{4}+n R\left(\xi, t_{n}\right)\right\} d \theta .
\end{aligned}
$$

Now we choose $\alpha$ such that $|R(\xi, t)| \leq a_{4}(\xi) t^{4} / 2$ for all $\xi \in[0, a]$ and $t \in[0, \alpha]$. This choice is possible since $|R(\xi, t)| \leq C|t|^{5}$ and $a_{4}(\xi)>c$ for all $\xi \leq a$. Then $\left|n R\left(\xi, t_{n}\right)\right| \leq \theta^{4} / 2$ and the modulus of the integrand in (8.1) is bounded by

$$
C \theta \exp \left\{-\frac{n^{1 / 4} a_{3}(\xi)}{\sqrt{2} a_{4}(\xi)^{3 / 4}} \theta^{3}-\frac{\theta^{4}}{2}\right\} \leq C \dot{\theta} \exp \left\{-C^{\prime} \theta^{3}-\frac{\theta^{4}}{2}\right\}
$$

if $\xi \in\left[0, n^{-3 / 4}\right]$ (we used $\left|v(\xi)+e^{-i \pi / 4} t_{n}\right| \leq C \theta$ ).

We proceed as in Theorem 5.5, we rewrite the decomposition of $G(z(\xi, t))$

$$
G(z(\xi, t))=\left(v(\xi)+e^{-i \pi / 4} t\right)^{-1} H(z(\xi, t))+\left(v(\xi)+e^{-i \pi / 4} t\right) K(z(\xi, t)) .
$$

Using this decomposition and the one of the remainder term $R$ in its real and imaginary parts, the integral in (8.1) can be written as

$$
\begin{aligned}
\int_{0}^{\alpha_{n}} \exp & \left\{-\frac{n^{1 / 4} a_{3}(\xi)}{\sqrt{2} a_{4}(\xi)^{3 / 4}} \theta^{3}-\theta^{4}+n R_{0}\left(\xi, t_{n}\right)\right\} z_{n}^{-1} \\
& \times \exp \left\{-i \frac{n^{1 / 2} a_{2}(\xi)}{a_{4}(\xi)^{1 / 2}} \theta^{2}-i \frac{n^{1 / 4} a_{3}(\xi)}{\sqrt{2} a_{4}(\xi)^{3 / 4}} \theta^{3}+i n R_{1}\left(\xi, t_{n}\right)\right\} \\
& \times\left\{\left(v(\xi)+e^{-i \pi / 4} t_{n}\right)^{2} H\left(z_{n}\right)+\left(v(\xi)+e^{-i \pi / 4} t_{n}\right)^{4} K\left(z_{n}\right)\right\} d \theta .
\end{aligned}
$$

Let $M=\exp \left\{-n^{1 / 4} a_{3}(\xi) \theta^{3} /\left(\sqrt{2} a_{4}(\xi)^{3 / 4}\right)-\theta^{4}+n R_{0}\left(\xi, t_{n}\right)\right\}, \tilde{f_{n}}(\theta, \xi)$ be the integrand, and $i A_{n}=i A(n, \xi, \theta)$ be the function in the exponential in $\widetilde{f_{n}}(\theta, \xi) / M$. Explicit computation shows that $\tilde{f_{n}}(\theta, \xi) / M$ can be written as

$$
\begin{aligned}
& \left\{\cos \left(A_{n}\right)\left(v(\xi)^{2}+\sqrt{2} v(\xi) t_{n}\right) H_{0}\left(z_{n}\right)\right. \\
& \left.\quad+\sin \left(A_{n}\right)\left(\sqrt{2} v(\xi) t_{n}+t_{n}^{2}\right) H_{0}\left(z_{n}\right)+o\left(v(\xi)^{2}\right)+o\left(t_{n}^{2}\right)\right\} \\
& \quad+i\left\{-\cos \left(A_{n}\right)\left(\sqrt{2} v(\xi) t_{n}+t_{n}^{2}\right) H_{0}\left(z_{n}\right)\right. \\
& \left.\quad+\sin \left(A_{n}\right)\left(v(\xi)^{2} \sqrt{2}+v(\xi) t_{n}\right) H_{0}\left(z_{n}\right)+o\left(v(\xi)^{2}\right)+o\left(t_{n}^{2}\right)\right\}
\end{aligned}
$$

where, as in the rest of the section, $o\left(v(\xi)^{2}\right)$ and $o\left(t_{n}^{2}\right)$ are uniform with respect to $\xi \in\left[0, n^{-3 / 4}\right]$. 
We extract the factor $n^{-1 / 2}$ from the integrand: thus the exponent of the $n$ outside of the integral is $-3 / 4$ as predicted by local estimates. Moreover, $\sqrt{n} v(\xi)^{2}, \sqrt{n} v(\xi) t_{n}$ and $\sqrt{n} t_{n}^{2}$ are bounded since $v(\xi) \sim \xi^{1 / 3}$ and $\xi \in\left[0, n^{-3 / 4}\right]$. We can write $\mathbf{A}$ as

$$
\begin{aligned}
& \frac{2 \sqrt{2} e^{n \phi(\xi)}}{\pi \sqrt[4]{a_{4}(\xi)} n^{3 / 4}} \int_{0}^{\alpha_{n}} \exp \left\{-\frac{n^{1 / 4} a_{3}(\xi)}{\sqrt{2} a_{4}(\xi)^{3 / 4}} \theta^{3}-\theta^{4}+n R_{0}\left(\xi, t_{n}\right)\right\} \\
& \quad \times\left(1+o\left(v(\xi)^{2}\right)+o\left(t_{n}^{2}\right)\right) \\
& \quad \times\left\{\cos \left(A_{n}\right)\left(\sqrt{n} v(\xi)^{2}+2 \sqrt{2 n} v(\xi) t_{n}+\sqrt{n} t_{n}^{2}\right) H_{0}\left(z_{n}\right)\right. \\
& \left.\quad+\sin \left(A_{n}\right)\left(\sqrt{n} t_{n}^{2}-\sqrt{n} v(\xi)^{2}\right) H_{0}\left(z_{n}\right)+\sqrt{n} o\left(v(\xi)^{2}\right)+\sqrt{n} o\left(t_{n}^{2}\right)\right\} d \theta .
\end{aligned}
$$

In order to apply Theorem 2.4, if $f_{n}(\theta, \xi)$ is the integrand in (8.3), we have to evaluate its uniform asymptotic $h_{n}(\theta, \xi)$. Using the asymptotic values of $a_{i}(\xi)$ and $v(\xi)$, and observing that $H\left(z_{n}\right)$ tends to $\sqrt{2}$ as $n$ tends to infinity,

$$
\begin{aligned}
h_{n}(\theta, \xi)= & \sqrt{2} e^{-2^{4 / 3} n^{1 / 4} \xi^{1 / 3} \theta^{3}-\theta^{4}}\left\{\cos \left(3 \cdot 2^{2 / 3} n^{1 / 2} \xi^{2 / 3} \theta^{2}-2^{4 / 3} n^{1 / 4} \xi^{1 / 3} \theta^{3}\right)\right. \\
& \times\left(2^{-1 / 3} n^{1 / 2} \xi^{2 / 3}+2^{4 / 3} n^{1 / 4} \xi^{1 / 3} \theta+\theta^{2}\right) \\
& \left.+\sin \left(3 \cdot 2^{2 / 3} n^{1 / 2} \xi^{2 / 3} \theta^{2}-2^{4 / 3} n^{1 / 4} \xi^{1 / 3} \theta^{3}\right)\left(\theta^{2}-2^{-1 / 3} n^{1 / 2} \xi^{2 / 3}\right)\right\} .
\end{aligned}
$$

The hypotheses of Theorem 2.4 are satisfied choosing

$$
g(\theta)=C e^{-C \theta^{3}-\theta^{4} / 2}\left(1+\theta+\theta^{2}\right) .
$$

Now we put $\delta:=n^{1 / 4} \xi^{1 / 3}$ : clearly $\delta \in[0,1]$ and the requirement that

$$
\left|\int_{\mathbb{R}^{+}} h_{n}(\theta, \xi) d \theta\right| \geq c>0
$$

for some $c$ and for every $\xi \in\left[0, n^{-3 / 4}\right]$, is equivalent to $I(\delta)>c$ for every $\delta \in[0,1]$. By numerical computation performed with Derive this appears obvious.

Hence by Theorem 2.4

$$
\mathrm{A} \stackrel{n}{\sim} \frac{4}{\pi} I\left(n^{1 / 4} \xi^{1 / 3}\right) e^{n \phi(\xi)} n^{-3 / 4}
$$

and this is the uniform asymptotic estimate for the $p^{(2 n)}(2 k, 0)$ since by Lemma 8.1 $|\mathbf{B} / \mathbf{A}|$ tends to zero uniformly with respect to $\xi \in\left[0, n^{-3 / 4}\right]$.

As in Theorem 5.5 one proves the statement for $\xi \in\left[0, n^{-3 / 4-\varepsilon}\right]$, noting that $I(0)=\int_{0}^{+\infty} \theta^{2} e^{-\theta^{4}} d \theta=\pi \sqrt{2} / 4 \Gamma(1 / 4)$. Observe that this last result can be obtained under the weaker assumption that $n^{1 / 4} \xi^{1 / 3} \rightarrow 0$ uniformly with respect to $\xi$. 


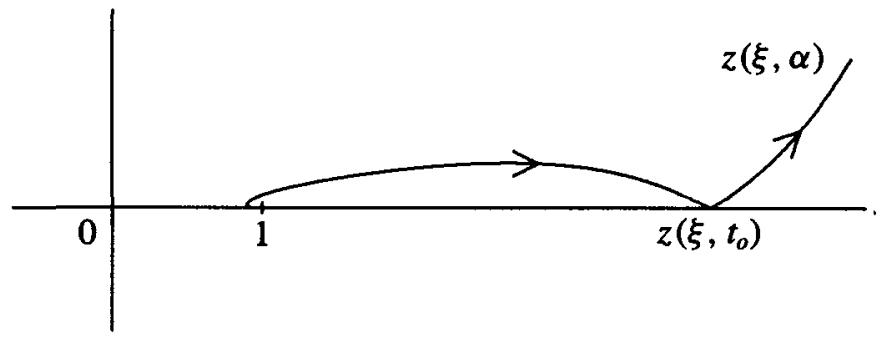

FIGURE 8. The curve $\gamma_{\left.\right|_{\mid l \in\{0, \alpha]}}$.

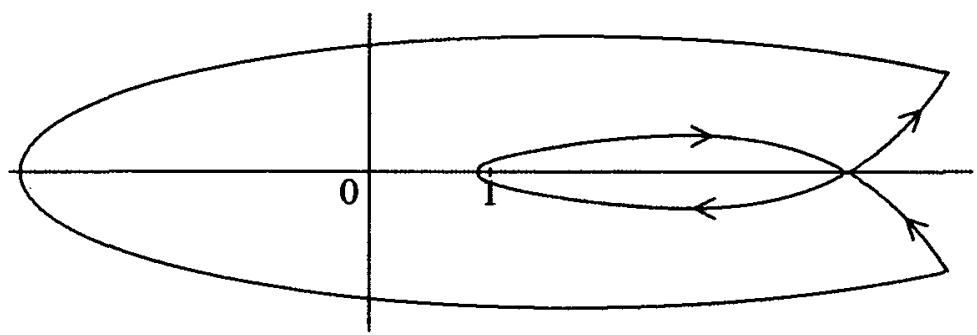

FIGURE 9. The curve of integration in the case $\xi \geq n^{-3 / 4}$.

\section{Estimates along the $x$-axis: the case $\xi \in\left[n^{-3 / 4}, a\right]$}

We observe that since in this case we have to use substitution (a), the curve of integration we used so far does not fit. In fact in the exponential, $\theta^{2}$ would have a pure imaginary coefficient. Hence we are forced to seek another solution, that is, a curve of integration made of more pieces: the curve $\gamma_{1}$ is $z(\xi, t)=1-\left(v(\xi)+e^{-i \pi / 3} t\right)^{4}$ if $0 \leq t \leq t_{o}, z(\xi, t)=1-\left(v(\xi)+e^{i \pi / 3} t\right)^{4}$ if $t_{o} \leq t \leq \alpha$, plus the conjugate of these two curves; while $\gamma_{2}$ is a circular arc which makes the whole connected. Figure 8 shows how these curves appear. A sketch for the curve of integration is shown in Figure 9.

As we already remarked, by symmetry $\mathbf{A}$, the integral over $\gamma_{1}$, is equal to

$$
\frac{1}{\pi} \operatorname{Im} \int_{0}^{\alpha} \frac{G(z(\xi, t))}{z(\xi, t)} \exp \left\{n \bar{\Psi}_{\xi}(t)\right\}\left[-4 e^{i \beta(t)}\left(v(\xi)+e^{i \beta(t)} t\right)^{3}\right] d t,
$$

where $\beta(t)=-\pi / 3$ if $t \in\left[0, t_{0}\right], \beta(t)=\pi / 3$ if $t \in\left(t_{0}, \alpha\right]$ and $t_{0}=2 /(\sqrt{3}-1) v(\xi)$. Thus we have to estimate

$$
\begin{aligned}
-\frac{4}{\pi} & \operatorname{Im} \int_{0}^{\alpha} \frac{G(z(\xi, t))}{z(\xi, t)} e^{n \phi(\xi)+n e^{-2 \pi / 3} a_{2}(\xi) t^{2}-n a_{3}(\xi) t^{3}+n e^{-4 \pi / 3} a_{4}(\xi) t^{4}+n R(\xi, t)} \\
& \times e^{-i \pi / 3}\left(v(\xi)+e^{-i \pi / 3} t\right)^{3} \mathbb{1}_{\left[0, t_{o}\right]}(t)+e^{i \pi / 3}\left(v(\xi)+e^{i \pi / 3} t\right)^{3} \mathbb{1}_{\left[t_{o}, \alpha\right]}(t) d t .
\end{aligned}
$$

Perform the change of variable $\theta=\sqrt{n a_{2}(\xi)} t$ and write $t_{n}$ for $\theta / \sqrt{n a_{2}(\xi)}$. Choose $\alpha$ such that $|R(\xi, t)| \leq a_{4}(\xi) t^{4} / 4$ for all $\xi \leq a$ and $|t| \leq \alpha$. Moreover in order to 
obtain $|z(\xi, \alpha)| \geq 1+\varepsilon_{o}$ for some $\varepsilon_{o}$, we let $\alpha>\max _{\xi} t_{o}=2 /(\sqrt{3}-1) v(a)$, which is surely true if $a$ is sufficiently small.

We distinguish two subcases: $\xi \in\left[n^{-3 / 4}, n^{-3 / 4+\varepsilon}\right]$ and $\xi \in\left[n^{-3 / 4+\varepsilon}, a\right]$.

THEOREM 9.1. For all $\varepsilon>0$ and for sufficiently small $a$, uniformly with respect to $\xi \in\left[n^{-3 / 4+\varepsilon}, a\right]$,

$$
p^{(2 n)}(2 k, 0) \stackrel{n}{\sim} \frac{4 v(\xi)^{3} G\left(z_{o}(\xi)\right)}{\pi z_{o}(\xi) \sqrt{a_{2}(\xi)}} e^{n \phi(\xi)} n^{-1 / 2} \int_{0}^{+\infty} e^{-\theta^{2} / 2} \cos \left(\frac{\pi}{6}-\frac{\sqrt{3}}{2} \theta^{2}\right) d \theta .
$$

ProOF. We apply Theorem 2.3, observing that the modulus of the integrand is dominated by $C(1+\theta)^{3} e^{\theta^{2} / 2-C \theta^{3}-C \theta^{4}}$, for every $\theta \geq 0, \xi \in\left[n^{-3 / 4+\varepsilon}, a\right]$ and $n$. Moreover, the integrand converges pointwise, uniformly with respect to $\xi \in\left[n^{-3 / 4+\varepsilon}, a\right]$, to $e^{-i \pi / 3} e^{e^{-2 \pi i / 3} \theta^{2}}$. Negligibility of the integral on $\gamma_{2}$ is shown as in the preceding cases.

THEOREM 9.2. For all $\varepsilon>0$, uniformly with respect to $\xi \in\left[n^{-3 / 4}, n^{-3 / 4+\varepsilon}\right]$,

$$
p^{(2 n)}(2 k, 0) \stackrel{n}{\sim} \frac{2^{1 / 6} G\left(z_{o}(\xi)\right)}{\pi \sqrt{3}} I\left(n^{-1 / 2} \xi^{-2 / 3}\right) e^{n \phi(\xi)} \xi^{2 / 3} n^{-1 / 2},
$$

where $I(t)$ is an integral function, defined as follows:

$$
\begin{aligned}
I(t) & :=\int_{0}^{+\infty} \exp \left\{-\frac{\theta^{2}}{2}-\frac{2^{11 / 6} t}{6 \sqrt{3}} \theta^{3}-\frac{t^{2}}{9 \cdot 2^{7 / 3}} \theta^{4}\right\} \\
& \left\{\sqrt{3} \cos \left(-\frac{\sqrt{3} \theta^{2}}{2}+\frac{\sqrt{3} t^{2}}{9 \cdot 2^{7 / 3}} \theta^{4}\right)\left(1+\sqrt{3} 2^{-1 / 6} t \theta-(3 \sqrt{6})^{-1} t^{3} \theta^{3}\right)\right. \\
& \left.-\sin \left(-\frac{\sqrt{3} \theta^{2}}{2}+\frac{\sqrt{3} t^{2}}{9 \cdot 2^{7 / 3}} \theta^{4}\right)\left(1-\sqrt{3} 2^{-1 / 6} t \theta-2^{2 / 3} t^{2} \theta^{2}-(3 \sqrt{6})^{-1} t^{3} \theta^{3}\right)\right\} \\
& \left\{1_{\left[0, \frac{2^{7 / 6}}{\sqrt{3}-1}\right.} \frac{\sqrt{3}]}{(\theta)}-\mathbb{1}_{\left[\frac{2^{7 / 6}}{\sqrt{3}-1} \frac{\sqrt{3}}{t},+\infty\right)}(\theta)\right\} d \theta .
\end{aligned}
$$

PROOF. The substantial difference with the previous case is that we cannot find a pointwise limit for the integrand, but only a pointwise asymptotic estimate. Hence we apply Theorem 2.4, exactly as in the proof of Theorem 8.2 .

\section{Final remarks}

First, let us observe how one can obtain an estimate for the transition probabilities with odd time and space parameters. 
From the results on the $y$-axis we obtain also $p^{(2 n+1)}\left(y_{2 k+1}, o\right)$, thanks to (4.1), as we noted in Section 4.

The estimate of $p^{(2 n+1)}\left(x_{2 k+1}, o\right)$ requires further calculation, but it is not much different from what we did this far. We briefly point out what has to be done.

Let $G(z), F(z)$ and $\Psi_{\xi}(z)$ be as in Section 6, then

$$
\begin{aligned}
p^{(2 n+1)}\left(x_{2 k+1}, o\right) & =\frac{1}{2 \pi i} \oint \frac{G(z)}{z} \frac{F(z)}{\sqrt{z}} e^{n \Psi_{\xi}(z)} d z \\
& =\frac{1}{2 \pi i} \oint \frac{G(z)}{z} \frac{1+\sqrt{1-z}-\sqrt{2} \sqrt{1-z+\sqrt{1-z}}}{z} e^{n \psi_{\xi}(z)} d z .
\end{aligned}
$$

Thus, if $\xi \in[a, 1-c]$ the proof will differ from that of Theorem 6.2 because one should multiply and divide the integrand by $G\left(z_{o}(\xi)\right) F\left(z_{o}(\xi)\right) / z_{o}(\xi)^{2}$ instead of $G\left(z_{o}(\xi)\right) / z_{o}(\xi)$. The rest of the proof is completely analogous to that of Theorem 6.2. The same can be done in the case $\xi \geq n^{-3 / 4}$.

In the case $\xi \in\left[0, n^{-3 / 4}\right]$ one will need a decomposition (similar to the decomposition (5.7) of $G(z))$ of $F(z) \sqrt{z}$ :

$$
1+\sqrt{1-z}-\sqrt{2} \sqrt{1-z+\sqrt{1-z}}=1+\sqrt{1-z}-\sqrt[4]{1-z} J(z)+\sqrt[4]{(1-z)^{3}} L(z),
$$

where $J(z)$ and $L(z)$ are two holomorphic functions. Then

$$
\begin{aligned}
G(z)(1 & +\sqrt{1-z}-\sqrt{2} \sqrt{1-z+\sqrt{1-z}}) \\
= & (1-z)^{-1 / 4} H(z)+(1-z)^{1 / 4}(H(z)+K(z)) \\
& +(1-z)^{1 / 2}(J(z) K(z)+H(z) L(z)) \\
& +(1-z)^{3 / 4} K(z)+\text { holomorphic functions, }
\end{aligned}
$$

and one can proceed as in Theorem 6.2.

Moreover, by symmetry, translation invariance and reversibility from our estimates of $p^{(n)}\left(x_{k}, o\right)$ and $p^{(n)}\left(y_{k}, o\right)$ (for $\left.k>0\right)$ one derives uniform estimates for $p^{(n)}\left(x_{k}, x_{k_{1}}\right)$, $p^{(n)}\left(\left(k_{1}, k\right), x_{k_{1}}\right), p^{(n)}\left(x_{k_{1}},\left(k_{1}, k\right)\right)$ for $k, k_{1} \in \mathbb{Z}$.

We note that it is not possible to extend these estimates to uniform estimates for any starting and ending point, as we did in (3.3) for local estimates since the asymptotic we use (picked from [3, Section 6]) does not hold uniformly.

We now remark some particular features of the transition probabilities of the simple random walk on $C_{2}$ that appear after our calculations. We recall our estimates for $\xi$ converging to zero with a 'controlled' speed, and we stress the dependence on $\xi$. Uniformly with respect to $\xi \in\left[n^{-3 / 4+\varepsilon}, n^{-1 / 2-\varepsilon}\right]$,

$$
p^{(2 n)}(x, o) \stackrel{n}{\sim} \begin{cases}C e^{-n \xi^{2}} n^{-3 / 4} & \text { if } x=y_{2 k} \\ C^{\prime} e^{-c n \xi^{4 / 3}} n^{-1 / 2} \xi^{1 / 3} & \text { if } x=x_{2 k}\end{cases}
$$


It is worth noting that, these results show a different dependence on $\xi$ along the two axes. In particular this results in the impossibility of finding for the transition probabilities on $C_{2}$ a sub-Gaussian estimate. Sub-Gaussian estimates of transition probabilities have been studied on many graphs: by Jones [11] on the 2-dimensional Sierpiński graph, by Barlow and Bass [1] on the graphical Sierpiński carpet and more recently on rather general graphs by Grigor' yan and Telcs $[9,16]$. If $\xi=d(x, y) / n$, sub-Gaussian estimates have this expression:

$$
c_{1} n^{-\delta_{s} / 2} e^{-c_{2} n \xi^{\left.\delta_{w} / \delta_{w}-1\right)}} \leq p^{(n)}(x, y) \leq c_{3} n^{-\delta_{s} / 2} e^{-c_{4} n \xi^{\left.\delta_{w} / \delta_{w}-1\right)}}, \quad \forall x, y,
$$

for some positive constants $c_{1}, c_{2}, c_{3}, c_{4}$ and for sufficiently large $n$. By definition $\delta_{s}$ is called the spectral dimension (which appears also in local estimates) and $\delta_{w}$ is the walk dimension. These two dimensions are in typical cases related by the so-called 'Einstein relation': $\delta_{s} \delta_{w}=2 \delta_{f}$, where $\delta_{f}$ is the fractal dimension (see $\left.[14,15]\right)$. Since for $C_{2}, \delta_{s}=3 / 2$ and $\delta_{f}=2$, one would expect $\delta_{w}=8 / 3$. Nevertheless (10.1) shows that if $x=y_{2 k}$ and $y=o$ the estimate holds with $\delta_{w}=2$, while if $x=x_{2 k}$ and $y=o$ the estimate holds with $\delta_{w}=4$.

We finally observe that the method we used here to provide a uniform asymptotic estimate of transition probabilities has already been employed for homogeneous trees and free groups (see $[12,13,18])$. One of the aims of this paper was also shedding new light onto this method. It seems that one could use the described technique for more general graphs and random walks (provided the knowledge of the Green function). As we noted, the major difficulty appears to be the choice of a proper contour of integration, for which no recipe is known (we investigated only a restricted family of curves in our case).

A natural extension of our results could be the analogues for $d$-combs. However, technical difficulties increase notably already for $d=3$, since

$$
G_{3}(z)=\frac{3}{\sqrt{3\left(1-z^{2}\right)+2 \sqrt{1-z^{2}}+2 \sqrt{2} \sqrt{1-z^{2}+\sqrt{1-z^{2}}}}} .
$$

\section{Acknowledgement}

We are deeply grateful to Professor Wolfgang Woess for his unceasing encouragement which helped us to reach these results.

\section{References}

[1] M. T. Barlow and R. F. Bass, 'Random walks on graphical Sierpiński carpets', in: Random walks and discrete potential theory (Cortona, 1997), Sympos. Math. 39 (Cambridge Univ. Press, Cambridge, 1999) pp. 26-55. 
[2] E. A. Bender, 'Asymptotic method in enumeration', SIAM Rev. 4 (1974), 485-515.

[3] D. Bertacchi and F. Zucca, 'Equidistribution of random walks on spheres', J. Stat. Phys. 94 (1999), 91-111.

[4] D. I. Cartwright, 'Some examples of random walks on free products of discrete groups', Ann. Mat. Pura Appl. (4) 151 (1988), 1-15.

[5] D. I. Cartwright and P. M. Soardi, 'Random walks on free products, quotients and amalgams', Nagoya Math. J. 102 (1986), 163-180.

[6] _ 'A local limit theorem for random walks on the Cartesian product of discrete groups', Boll. Un. Mat. Ital. A (7) 1 (1987), 107-115.

[7] D. Cassi and S. Regina, 'Random walks on $d$-dimensional comb lattices', Modern Phys. Lett. B 6 (1992), 1397-1403.

[8] P. Gerl, 'Natural spanning trees of $\mathbb{Z}^{d}$ are recurrent', Discrete Math. 61 (1986), 333-336.

[9] A. Grigor'yan and A. Telcs, 'Sub-Gaussian estimates of heat kernels on infinite graphs', Duke Math. J. 109 (2001), 451-510.

[10] S. Havlin and G. H. Weiss, 'Some properties of a random walk on a comb structure', Phys. A 134 (1986), 474-482.

[11] O. D. Jones, 'Transition probabilities for the simple random walk on the Sierpiński graph', Stochastic Process. Appl. 61 (1996), 4-69.

[12] S. P. Lalley, 'Saddlepoint approximations and space-time Martin boundary for nearest neighbour random walk on a homogeneous tree', J. Theoret. Probab. 4(1991), 701-723.

[13] _ , 'Finite range random walks on free groups and homogeneous trees', Ann. Probab. 21 (1993), 2087-2130.

[14] A. Telcs, 'Spectra of graphs and fractal dimensions. I', Probab. Theory Related Fields 85 (1990), $489-497$.

[15] _ - 'Spectra of graphs and fractal dimensions. II', Probab. Theory Related Fields 8 (1995), 77-96.

[16] _ 'Local sub-Gaussian estimates on graphs, the strongly recurrent case', Electron. J. Probab. $6(2001), 33$.

[17] W. Woess, 'Nearest neighbour random walks on free product of discrete groups', Boll. Un. Mat. Ital. $B(6) 5$ (1986), 961-982.

[18] - Random walks on infinite graphs and groups, Cambridge Tracts in Mathematics 138 (Cambridge Univ. Press, Cambridge, 2000).

Università di Milano-Bicocca

Dipartimento di Matematica e Applicazioni

Via Bicocca degli Arcimboldi 8

20126 Milano

Italy

e-mail: bertacchi@matapp.unimib.it
Politecnico di Milano Dipartimento di Matematica Piazza Leonardo da Vinci 32 20133 Milano Italy

e-mail: zucca@mate.polimi.it 
